\title{
OPTIMAL HORIZONS FOR INFLATION TARGETING
}

\author{
Nicoletta Batini \\ and \\ Edward Nelson \\ Bank of England \\ January 1999 \\ Preliminary and Incomplete \\ Please do not quote without permission
}

The views expressed in this paper do not necessarily reflect those of the Bank of England. 


\section{List of contents}

page

Abstract

3

Introduction

4

1. Objective function and models 6

1.a The objective function 6

1.b The models 6

2. Optimal Policy Horizons (OPHs) 13

2.a Numerical derivation of OPHs $\quad 14$

2.a.1 Results for different sets of weights 22

3. Optimal Feedback Horizons (OFHs) 24

3.a Numerical derivation of OFHs 28

3.b A comparison of OFHs and $\mathrm{PHs} / \mathrm{OPHs} \quad 36$

4. Conclusions 48

Data Appendix $\quad 52$

Technical Appendix ( Solution Methods $\quad 55$

$\begin{array}{ll}\text { References } & 57\end{array}$ 



\begin{abstract}
In this paper we investigate the problem of selecting an optimal horizon for inflation targeting in the United Kingdom. Since there are several ways to think about an optimal horizon, we look at optimal horizons for each of these interpretations. Also, to see whether our results are robust in the face of model uncertainty, we derive results for a set of models - rather than just one model — with varying structural and dynamic characteristics. Optimal horizons for each model are derived numerically.
\end{abstract}




\section{Introduction}

Much recent analysis suggests that inflation targets are best pursued by targeting

future inflation (Bowen (1995), Haldane (1995), King (1996), Nixon and Hall (1996), Svensson $(1996,1997)$. Current inflation is largely predetermined by existing contracts and it is affected by monetary policy only with a lag. In these circumstances, the authorities can affect future inflation by acting in a pre-emptive fashion. The way this is usually done in practice involves 'targeting' the conditional forecast of inflation, that is, the inflation rate expected to prevail in the future given presently available information. Using the conditional forecast for inflation as an intermediate target ensures that the policy rule recognises the lags in the effect of monetary policy, thus avoiding the problem posed by targeting current inflation.

One crucial issue with conditional forecast targeting is that of choosing the best forecast to use as an intermediate target, that is, the appropriate value of $k$ when the intermediate target is expected inflation $k$-periods-ahead.

This issue is non-trivial, because the nature of the optimal forecast horizon will differ according to the role assigned to the intermediate target in the policy process. If the policymakers operate via an explicit policy rule that involves moving the interest rate (the operating variable) to correct deviations of expected inflation (the intermediate variable) from its target value, that is, via a rule like:

$R_{t}=\psi_{p}\left(\mathrm{E}_{t-1} \pi_{t+k}-\pi^{T A R}\right)$

where $R_{t}$ is the short-term nominal interest rate, $\pi_{t}$ is inflation, $\pi^{T A R}$ is the inflation target and $\mathrm{E}_{t-1}$ is the expectation operator at time $t-1$, then expected inflation deviations from target become an explicit feedback variable. In this case, the best 
$k$-period-ahead forecast of inflation to use as an intermediate target will be the one for which it is most efficient to correct deviations. In mathematical terms, the optimal forecast horizon will be the $k$ associated with (1) that minimises the costs of inflation stabilisation for a given social welfare function.

If policymakers instead use an implicit policy rule that involves reacting to a wider set of variables than just deviations of expected inflation from its target (as is done in practice in the UK), then the latter will be neither an explicit nor a unique intermediate feedback variable. In this case, the horizon chosen for the forecast of inflation to use as the intermediate target is perhaps best interpreted as the time in the future at which inflation should be brought in line with the target; so that, for example, using an intermediate target on expected inflation $k$ quarters ahead means inflation should be on target in $k$ quarters' time. If so, then the best $k$-period-ahead forecast of inflation to use as an intermediate target may be that corresponding to the time in the future when, under an optimal policy rule, inflation is back on target after a shock today. Such an optimal horizon can be derived by standard optimisation methods.

In summary, there are various ways to think of an 'optimal horizon' for inflation targeting. One way is to think of it as the (best) horizon at which the authorities should form the forecast for inflation on which to feed back. A second way is to think of it as the time from which inflation should be on target in the future when the authorities aim at minimising the costs of disinflation (i.e., use an optimal control rule for the instrument). In what follows, we refer to the first kind of horizon as the optimal feedback horizon and to the second kind the optimal policy horizon.

This paper investigates what the optimal horizon for the United Kingdom might be under either of the above definitions. Since the results may well hinge on the features of the model used for the investigation, the paper derives parallel results for two different models of the UK economy: a vector autoregression (VAR) estimated on quarterly data; and several variants of a small calibrated forward-looking model 
The paper is organised as follows. In Section 1 we describe the policymaker's objective function and the macroeconomic models that we employ. In Section 2 we obtain optimal policy horizons for each model numerically, and then discuss the results. In Section 3 we focus attention on optimal feedback horizons. Concluding remarks follow. A Data Appendix describes the definitions and time series properties of the data used in estimating our VAR, and a Technical Appendix describes the solution methods employed in Section 2 for the numerical derivation of the optimal policy rules.

\section{Objective function and models}

1.a The objective function

We assume that policymakers dislike inflation deviations from target and output deviations from potential. For convenience, throughout this paper, these preferences with are represented by a quadratic welfare loss function. In the optimisation exercises used to derive optimal policy horizons, this is the function that is being minimised. Second, when we derive optimal feedback horizons by comparing the performance of rules like (1) with various $k \mathrm{~s}$, this welfare function is used to compute welfare losses in a way comparable across all simulations. The welfare loss function chosen is:

$L_{t}=\mathrm{E}_{t} \sum_{j=0}^{\infty}\left(\lambda \lambda^{j}\left[\pi_{t+j}-\pi_{t+j}{ }^{T A R}\right)^{2}+\lambda y\left(y_{t+j}-y_{t+j}{ }^{T}\right)^{2}\right]$

where (is the discount factor, $\pi_{t}$ is quarterly inflation, $\pi_{t}^{T A R}$ is target inflation, $y_{t}$ is $\log$

output, $y_{t}^{T}$ is $\log$ capacity output, and where $\lambda$ (and $\lambda_{y}$ denote the weights assigned to inflation deviations from target and output deviations from trend, respectively. 
As a benchmark, we set $\left(=0.99\right.$, and $\lambda\left(=\lambda_{y}=0.5\right.$, so that inflation and output variability are penalised equally, although we also experiment with different sets of weights.

\section{1.b The models}

To explore the optimal horizon problem, we look at two models: a vector autoregression (VAR) estimated on quarterly data; and several variants of a small calibrated forward-looking model (Batini and Haldane [1999]). They are described below.

\section{A VAR model}

Our first model is a one-lag vector autoregression (VAR) with a linear trend, estimated over the period 1981 Q1-1998 Q1. There are four endogenous variables in the VAR: $\log$ output $\left(y_{t}\right)$; the deviation of twelve-month RPIX inflation from the inflation target $\left(\pi_{t}^{D E V}\right)$; the change in the nominal exchange rate $\left(\Delta e_{t}\right)$; and the nominal interest rate (interbank lending rate), measured as a fraction $\left(R_{t}\right){ }^{1}$

To capture shifts in monetary policy regime during the estimation period, we also include two dummy variables: a dummy for the 1990 Q4 - 1992 Q3 period of UK membership of the Exchange Rate Mechanism $\left(D E R M_{t}\right)$; and $D 924_{t}$, a dummy for the new inflation targeting regime that started in October $1992 .^{2}$

Disregarding the constant, the trend, and the policy shift terms, the VAR may be written as:

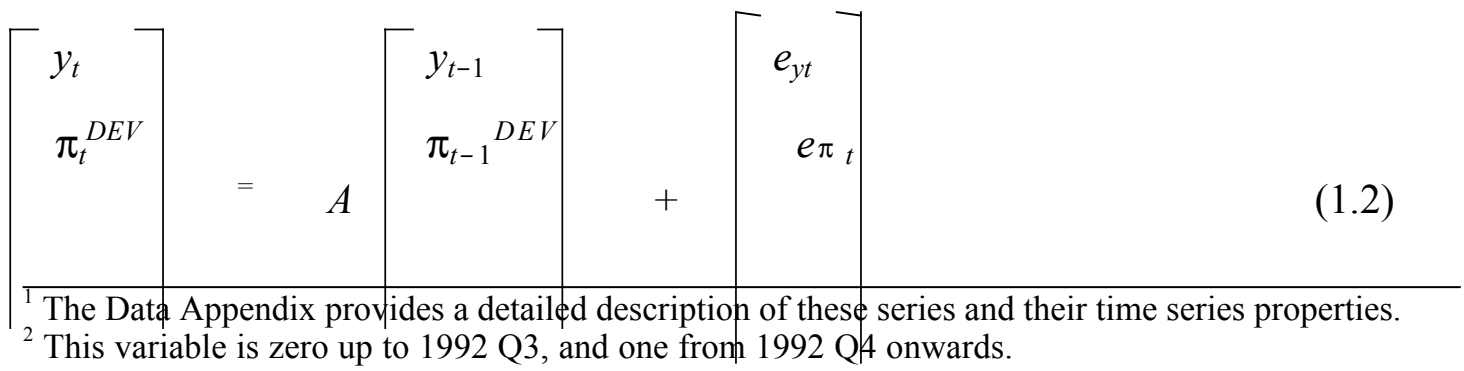




$\begin{array}{lrr}\Delta e_{t} & \Delta e_{t-1} & e_{e t} \\ \left\llcorner^{R_{t}}\right\lrcorner & \left\llcorner^{R_{t-1}}\right\lrcorner & e_{R t}\end{array}$

where $A$ is a 4 (4 matrix of estimated coefficients and $\left(e_{y t}, e \pi_{t}, e_{e t}, e_{R t}\right)$ are the equations' disturbances. Estimates of this system are reported in Table 1. The lag length of one quarter is not rejected by a $\chi^{2}$ test against the alternative of a two-lag $\operatorname{VAR}(p-$ value $=0.12)$

Looking at Table 1, a few points regarding the dynamic properties of the system emerge. First, although the estimated coefficient on the interest rate in the output regression is quite small (-0.0908), and hence suggests a negligible initial impact of monetary policy on real variables, the estimated coefficient on the lagged dependent variable in the output equation is large, implying that the long-run coefficient on the interest rate in the output equation is much larger (at $-0.0908 /(1-0.9321)=-1.3373$ percent).

Secondly, in line with economic intuition, output has a significant positive coefficient in the inflation equation; and so interest rates have a negative effect on inflation, apparently via a conventional output gap channel. ${ }^{3}$

Finally, the estimated equation for $R_{t}$ looks like a Taylor-type policy rule, with the interest rate responding positively both to lagged output (long-run coefficient 0.10 ) and to inflation (long-run coefficient 0.91 , not significantly below unity). In addition, the coefficient on the lagged dependent variable (0.58) suggests a strong tendency by policymakers to smooth interest rates; and the coefficient on the exchange rate change (equal to -0.042) suggests that the interest rate responds negatively to exchange rate

\footnotetext{
Structural interpretations of this kind have to be subject to the caveat that unrestricted VARs are
} 
depreciations, as one would expect. ${ }^{4}$ The dummy variables for regime shifts enter the $R_{t}$ equation significantly.

${ }^{\overline{4}}$ Notice that no variables enter the exchange rate equation itself significantly, indicating that a random 


\begin{tabular}{|c|c|c|c|c|}
\hline \multicolumn{5}{|c|}{$\begin{array}{c}\text { Table 1: } \text { VAR } \text { :stimates } \\
\text { Sample period: } 1981 \text { ì1 - } 1998 \text { Q1 }\end{array}$} \\
\hline & $y_{t}$ & $\pi^{D E V}{ }_{t}$ & $\Delta e_{t}$ & $R_{t}$ \\
\hline$y_{t-1}$ & $\begin{array}{r}0.9321 \\
(0.0464)\end{array}$ & $\begin{array}{l}0.1250 \\
(0.0575)\end{array}$ & $\begin{array}{r}-0.1583 \\
(0.3273)\end{array}$ & $\begin{array}{l}0.0444 \\
(0.0782)\end{array}$ \\
\hline$\pi_{t-1}^{D E V}$ & $\begin{array}{r}-0.0926 \\
(0.0731)\end{array}$ & $\begin{array}{c}0.8559 \\
(0.0905)\end{array}$ & $\begin{array}{r}0.3544 \\
(0.5150)\end{array}$ & $\begin{array}{r}0.3867 \\
(0.1231)\end{array}$ \\
\hline$\Delta e_{t-1}$ & $\begin{array}{r}-0.0029 \\
(0.0175)\end{array}$ & $\begin{array}{r}-0.0023 \\
(0.0217)\end{array}$ & $\begin{array}{r}0.1526 \\
(0.1234)\end{array}$ & $\begin{array}{r}-0.0416 \\
(0.0295)\end{array}$ \\
\hline$R_{t-1}$ & $\begin{array}{r}-0.0908 \\
(0.0529)\end{array}$ & $\begin{array}{c}0.0336 \\
(0.0655)\end{array}$ & $\begin{array}{r}-0.0438 \\
(0.3731)\end{array}$ & $\begin{array}{l}0.5759 \\
(0.0892)\end{array}$ \\
\hline constant & $\begin{array}{r}0.7821 \\
(0.5177)\end{array}$ & $\begin{array}{r}-1.4072 \\
(0.6409)\end{array}$ & $\begin{array}{l}1.7167 \\
(3.6487)\end{array}$ & $\begin{array}{r}-0.4786 \\
(0.8722)\end{array}$ \\
\hline $\begin{array}{l}\text { time } \\
\text { trend }\end{array}$ & $\begin{array}{r}0.00046 \\
(0.00040)\end{array}$ & $\begin{array}{r}-0.00091 \\
(0.00049)\end{array}$ & $\begin{array}{l}0.002477 \\
(0.00280)\end{array}$ & $\begin{array}{c}0.000287 \\
(0.00067)\end{array}$ \\
\hline$D E R M_{t}$ & $\begin{array}{r}-0.00891 \\
(0.00563) \\
\end{array}$ & $\begin{array}{c}0.0091 \\
(0.0070)\end{array}$ & $\begin{array}{r}-0.0509 \\
(0.0397)\end{array}$ & $\begin{array}{r}-0.0300 \\
(0.0095)\end{array}$ \\
\hline$D 924_{t}$ & $\begin{array}{r}-0.0091 \\
(0.0073)\end{array}$ & $\begin{array}{l}0.0108 \\
(0.0090)\end{array}$ & $\begin{array}{r}-0.0573 \\
(0.0515)\end{array}$ & $\begin{array}{r}-0.0422 \\
(0.0123)\end{array}$ \\
\hline $\mathrm{R}^{2}$ & 0.9984 & 0.8880 & 0.1017 & 0.9259 \\
\hline S.E.E. & 0.0052 & 0.0064 & 0.0364 & 0.0087 \\
\hline $\begin{array}{c}\text { S.D. } \\
\text { dependent }\end{array}$ & 0.1212 & 0.0181 & 0.0364 & 0.0303 \\
\hline \multicolumn{5}{|c|}{ Note: Standard errors in parentheses } \\
\hline
\end{tabular}


In this paper we subject the VAR to hypothetical policy rules different from the estimated one. This is only possible if we identify the VAR model's responses to shocks. We do this by means of a Cholesky decomposition, where the equation disturbances are assumed to follow the causal ordering $\left(e_{y t}, e_{e t}, e \pi_{t}, e_{R t}\right)$. Under this identification scheme, no variable beside the interest rate responds contemporaneously to the interest rate shock.

\section{A small open-economy linear RE model}

Our second model is a small open-economy linear RE model calibrated for the UK (Batini and Haldane [1999], henceforth, BH). This model differs from the VAR in two major respects: it imposes somewhat more structure; and it is forward-looking. Setting capacity output and foreign variables to constants and suppressing the intercept terms in each equation, the model ( with all variables now expressed as deviations from equilibrium ( can be expressed as:

$$
\begin{aligned}
& y_{t}=\alpha_{1} y_{t-1}+\alpha_{2} \mathrm{E}_{t} y_{t+1}+\alpha_{3}\left[R_{t-1}-\mathrm{E}_{t-1} \pi_{t}\right]+\alpha_{4} q_{t-1}+\varepsilon_{I S t-1} \\
& \pi_{t}=\chi_{0} \mathrm{E}_{t} \pi_{t+1}+\left(1-\chi_{0}\right) \pi_{t-1}+\chi_{1}\left[y_{t}+y_{t-1}\right]+\chi_{2}\left[\left(1-\chi_{0}\right) \Delta q_{t}-\chi_{0} \mathrm{E}_{t} \Delta q_{t+1}\right]+\varepsilon \pi_{t} \\
& \mathrm{E}_{t} \Delta q_{t+1}+\mathrm{E}_{t} \pi_{t+1}=R_{t}+\varepsilon_{U I P t}
\end{aligned}
$$

where $y_{t}$ is quarterly log output, $\pi_{t}$ is quarterly inflation, $R_{t}$ is defined as in the VAR, $q_{t}$ is the $\log$ of the real exchange rate, and $\varepsilon_{I S t}, \varepsilon \pi_{t}$, and $\varepsilon_{U I P t}$ are white-noise disturbances. Equation (1.3) is the model's IS curve, with real output depending negatively on the ex ante real interest rate and positively on the log of the real exchange rate. Equation (1.4) is an open-economy aggregate supply equation (more on this later); and (1.5) is a standard uncovered interest parity relationship with a stochastic risk premium.

Model (1.3)-(1.5) is forward-looking in three ways. There is the conventional forward-looking behaviour inherent in the UIP condition. But in addition, the output 
and inflation equations also have forward-looking elements, since they both include lead terms for the dependent variable on their right-hand sides. The parameters $\alpha_{1}, \alpha_{2}$, and $\chi_{0}$ jointly govern the overall degree of forward-looking behaviour in the model, and can be selected to obtain several models that are special cases of (1.3)-(1.5). When 0 $<\alpha_{1}<1$ and $\alpha_{2}=0$, for example, the IS equation is entirely backward-looking, whereas when $\alpha_{1}=0$ and $\alpha_{2}=1$, it is a forward-looking expectational IS curve, of a type that can be derived from a general equilibrium model. ${ }^{5}$ Similarly, when $\chi_{0}=1$, the aggregate supply equation (1.4) is simply an open-economy version of Taylor's (1980) staggered contracts model; when $\chi_{0}=0.5$, it is an open-economy analogue of Fuhrer and Moore's (1995) contracting model; and with $\chi_{0}=0$ it coincides with a fully backward-looking open-economy Phillips curve.

Given its flexible specification, we can consider different variants of model (1.3)-(1.5) when computing optimal policy horizons and optimal feedback horizons. We use three. Each variant uses a certain combination of the parameters $\alpha_{1}, \alpha_{2}$ and $\chi_{0}$, and thus is associated with a particular degree of overall forward-looking behaviour in the economy. More precisely:

BH Variant 1: $\alpha_{1}=0.8, \alpha_{2}=0$, and $\chi_{0}=0.2$. This variant of the model has a backward-looking IS function and a partially forward-looking AS equation (as in Fuhrer (1997)). This may be the most appropriate setting of the parameters if expectations are a moderately significant factor in wage/price setting decisions but not in household spending decisions.

$B H$ Variant 2: $\alpha_{1}=0, \alpha_{2}=1.0, \chi_{0}=0.2$. This second variant of the model has a forward-looking IS and a partially forward-looking AS equation. This variant provides insights about the consequences of relaxing the assumption that expectations do not enter the aggregate demand function. Note that while (as in the other BH variants) the exchange rate and the interest rate have direct effects on output only with 
a lag, monetary policy can nevertheless affect output today because its actions affect the expected value of output $\mathrm{E}_{t} y_{t+1}$ in equation (1.3).

BH Variant 3: $\alpha_{1}=0.8, \alpha_{2}=0, \chi_{0}=1.0$. This last variant of the model has a backward-looking IS and a fully forward-looking AS equation (corresponding to Taylor (1980) staggered price contracts). This variant provides an indication of the behaviour of the $\mathrm{BH}$ model in the case where inflation has no inherent inertia.

Throughout, we fix the remaining parameters of the model at values in line with prior empirical estimates on quarterly data, as in Batini and Haldane (1999), and we adopt the same specification of shock variances used in that paper. We assume that the shocks follow the ordering $\left(\varepsilon_{I S t-1}, \varepsilon_{U I P t}, \varepsilon \pi_{t}\right)$. 


\section{Optimal policy horizons (OPHs)}

If policymakers feed back implicitly on a wide set of variables rather than solely on deviations of expected inflation from its target (as occurs in practice in the UK), then the relevant horizon for inflation targeting is the time in the future at which inflation should be brought on target.

Defining the time at which inflation and the inflation target are to be in line in the future as the policy horizon $(\mathrm{PH})$, the optimal policy horizon $(\mathrm{OPH})$ is then the time at which it is least costly, for a given specification of the loss function, to bring inflation back to target after a shock. More intuitively, the OPH is the horizon-analogue of the optimal speed of disinflation. In practice, the $\mathrm{OPH}$ is given by 'the' time at which inflation is on target under an optimal rule, because, by definition, an optimal rule provides a path for the instrument which stabilises inflation at the minimum possible cost.

In what follows, we derive optimal policy horizons for each model. An important question is what criterion we should use for being 'on target'. Since inflation in the models tends not to settle exactly on a particular number in the wake of a shock, a point target of 2.5 is not very meaningful. Instead, we consider optimal policy horizons defined over target zones. Specifically, rather than thinking of an $\mathrm{OPH}$ as the time at which inflation settles back exactly on a point-target after an inflationary shock, we think of it as the time at which inflation returns to target and remains within a specified band around the target (a 'target zone'). This is not an argument for target zones rather than point targets, but a device to make model simulation experiments useful.

We look at three kind of zones: two absolute and a relative target zone. This gives us three operational definitions of an optimal policy horizon. The first one interprets an optimal policy horizon as the time $k$ periods ahead at which inflation has returned 
permanently to within a target zone of ( 1 percentage points, following a shock today; and the second one interprets it as the time $k$ periods ahead at which inflation has definitely returned to within a target zone $(0.05$ percentage points around target after a shock today. These 'absolute criteria' have the disadvantage of being sensitive to the size of the shock under investigation; on the other hand, these criteria are useful because they give a feel for how long it takes for inflation to be broadly on target under an optimal rule. The third target zone interprets the optimal policy horizon as the time $k$ at which $95 \%$ of the initial effect of the shock on inflation has been permanently eroded. This criterion has the advantage over the absolute criteria that it is insensitive to the choice of units for the initial shock: a $1 \%$ shock versus a onestandard-deviation shock, say. ${ }^{6}$ However, it may imply extremely long optimal policy horizons whenever the initial effect of the shock on inflation is small: this is because it requires returning to a target zone whose width is a fraction of a small initial effect - in effect, requiring a return to a point target. We denote OPHs under the first and second absolute criteria, by ' $k_{A 1}{ }^{*}$ ' and ' $k_{A 2}{ }^{*}$ ', respectively, and OPHs under the third, relative criterion, by ' $k_{R}{ }^{*}$ '.

\section{2.a Numerical results}

After solving the four models of Section 1 (the VAR, BH Variant 1, BH Variant 2 and BH Variant 3) via the method described in the Technical Appendix, we compute impulse responses for each model under the optimal rule. Figures 1 to 3 show the optimal paths of inflation associated with each model in the face of $1 \%$ shocks to aggregate demand, aggregate supply and the exchange rate. An optimal policy horizon is the time at which these paths return to and remain within the absolute and relative target zones. Table 2 gives optimal policy horizons for each model under the first absolute criterion, $k_{A 1} *$. Results in Table 2 show, trivially, that for all models, it is

\footnotetext{
${ }^{6}$ For example, if inflation takes ten quarters to settle within \pm 0.05 points of its initial value in response to a $1 \%$ shock, then $k=10$ by the aforementioned 0.05 points (second) absolute criterion. However, if a one-standard-deviation value of that shock is $2 \%$ and that value is used for the initial shock, then inflation fails the 0.05 points criterion at $k=10$. Instead, using our relative criterion, the optimal $k^{*}$ is 10 regardless of the size of the initial shock.
} 
optimal to disinflate any shock within one quarter at the latest. This is because under the optimal rule, in all cases inflation does not even exit, or reaches immediately, the (1 percent target zone following a $1 \%$ shock; results would differ had the shock been greater than $1 \%$, because the criterion is sensitive to the size of the shock.

Results in Tables 3-6 are more interesting. These tables give optimal policy horizons for each model under the two remaining definitions $\left(k_{A 2}{ }^{*}\right.$ and $\left.k_{R}{ }^{*}\right)$. We discuss each shock in turn.

Notice that Table 2 and the figures below relate to a baseline specification of the loss function (1.1) that imposes $\lambda_{e}=\lambda_{y}=0.5$; whereas Tables 3-6 present results also for different sets of weights. 
Table 2: OPHs ( All models ${ }^{a}$

\begin{tabular}{|l|c|c|c|c|}
\hline Models & BN VAR & BH Variant 1 & BH Variant 2 & BH Variant 3 \\
\hline & $k_{A 1}{ }^{*}$ & $k_{A 1}{ }^{*}$ & $k_{A 1}{ }^{*}$ & $k_{A 1}{ }^{*}$ \\
\hline AD Shock & 0 & 1 & 0 & 0 \\
\hline AS shock & 1 & 0 & 0 & 0 \\
\hline Exchange Rate Shock & 0 & 0 & 0 & 0 \\
\hline
\end{tabular}

(a): $k_{A 1} *$ refers to the number of periods, under the optimal rule, that it takes inflation to return permanently to within $+/-1$ percent of its initial value, following an initial 1 percent shock of the indicated type.

Table 3: OPHs ( $\mathrm{BN} \mathrm{VAR}{ }^{a}$

\begin{tabular}{|c|c|c|c|c|c|c|}
\hline & \multicolumn{6}{|c|}{ Value of $k^{*}$ for weight combination: } \\
\hline & \multicolumn{2}{|c|}{$\lambda\left(=\lambda_{y}=0.5\right.$} & \multicolumn{2}{|c|}{$\lambda_{F}=1.0, \lambda_{y}=0$} & \multicolumn{2}{|c|}{$\lambda\left(=0.5, \lambda_{y}=1.0\right.$} \\
\hline & $k_{A 2}{ }^{*}$ & $k_{R}^{*}$ & $k_{A 2} *$ & $k_{R}^{*}$ & $k_{A 2}{ }^{*}$ & $k_{R}^{*}$ \\
\hline AD shock & 10 & 16 & 10 & 11 & 20 & 11 \\
\hline AS shock & 16 & 16 & 15 & 15 & 16 & 16 \\
\hline Exch. rate shock & 0 & 18 & 0 & 17 & 0 & 18 \\
\hline \multicolumn{7}{|c|}{$\begin{array}{l}\text { (a): } k_{A 2} * \text { refers to the number of periods, under the optimal rule, that it takes inflation to return } \\
\text { permanently to within } 0.05 \text { percent of its initial value, following an initial } 1 \text { percent shock of the } \\
\text { indicated type; } k_{R} * \text { refers to the number of periods it takes for } 95 \text { percent of the initial effect of the } \\
\text { indicated shock to inflation to have been permanently eroded. }\end{array}$} \\
\hline
\end{tabular}


Table 4: OPHs ( BH Variant $1^{a}$

\begin{tabular}{|c|c|c|c|c|c|c|}
\hline & \multicolumn{5}{|c|}{ Value of $k^{*}$ for weight combination: } \\
\hline & \multicolumn{2}{|c|}{$\lambda=\lambda_{y}=0.5$} & \multicolumn{2}{c|}{$\lambda=1.0, \lambda_{y}=0$} & \multicolumn{2}{c|}{$\lambda=0.5, \lambda_{y}=1.0$} \\
\hline & $k_{A 2}{ }^{*}$ & $k_{R}{ }^{*}$ & $k_{A 2}{ }^{*}$ & $k_{R}{ }^{*}$ & $k_{A 2}{ }^{*}$ & $k_{R}{ }^{*}$ \\
\hline AD shock & 10 & 10 & $\infty$ & $\infty$ & 10 & 9 \\
\hline AS shock & 3 & 11 & 2 & $\infty$ & 3 & 10 \\
\hline Exch. rate shock & 13 & 20 & $\infty$ & $\infty$ & 14 & 15 \\
\hline
\end{tabular}

(a): BH Variant 1 has a backward-looking IS function and a mixed backward / forward-looking AS equation. $k_{A 2}$ * refers to the number of periods, under the optimal rule, that it takes inflation to return permanently to within 0.05 percent of its initial value, following an initial 1 percent shock of the indicated type; $k_{R} *$ refers to the number of periods it takes for 95 percent of the initial effect of the indicated shock to inflation to have been permanently eroded.

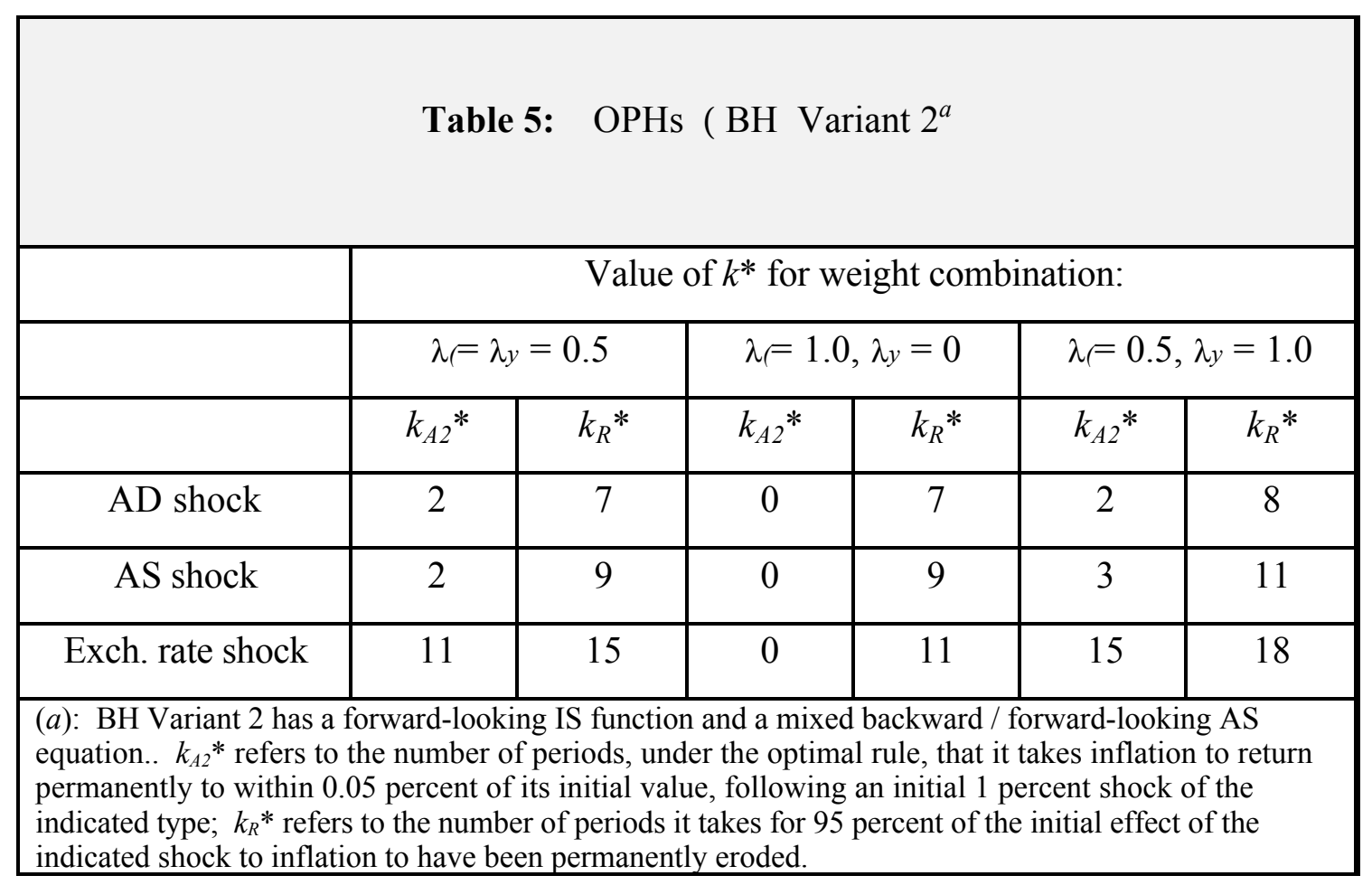


Table 6: OPHs ( BH Variant $3^{a}$

\begin{tabular}{|c|c|c|c|c|c|c|}
\hline & \multicolumn{6}{|c|}{ Value of $k^{*}$ for weight combination: } \\
\hline & \multicolumn{2}{|c|}{$\lambda\left(=\lambda_{y}=0.5\right.$} & \multicolumn{2}{c|}{$\lambda\left(=1.0, \lambda_{y}=0\right.$} & \multicolumn{2}{c|}{$\lambda=0.5, \lambda_{y}=1.0$} \\
\hline & $k_{A 2}{ }^{*}$ & $k_{R}{ }^{*}$ & $k_{A 2}{ }^{*}$ & $k_{R}{ }^{*}$ & $k_{A 2}{ }^{*}$ & $k_{R}{ }^{*}$ \\
\hline AD shock & 10 & 12 & 0 & 9 & 14 & 15 \\
\hline AS shock & 3 & 18 & 0 & 7 & 4 & 19 \\
\hline Exch. rate shock & 10 & 18 & 0 & 8 & 15 & 25 \\
\hline
\end{tabular}

(a): BH Variant 3 has a backward-looking IS function and a forward-looking AS equation. $k_{A 2}{ }^{*}$ refers to the number of periods, under the optimal rule, that it takes inflation to return permanently to within 0.05 percent of its initial value, following an initial 1 percent shock of the indicated type; $k_{R}{ }^{*}$ refers to the number of periods it takes for 95 percent of the initial effect of the indicated shock to inflation to have been permanently eroded.

\section{Aggregate demand shock}

Figure 1 (with the $x$-axis in calendar quarters, and the $y$-axis in percentage points deviations from base) shows the impulse responses of inflation for the four models described in Section 1, in the face of a temporary $1 \%$ shock to aggregate demand.

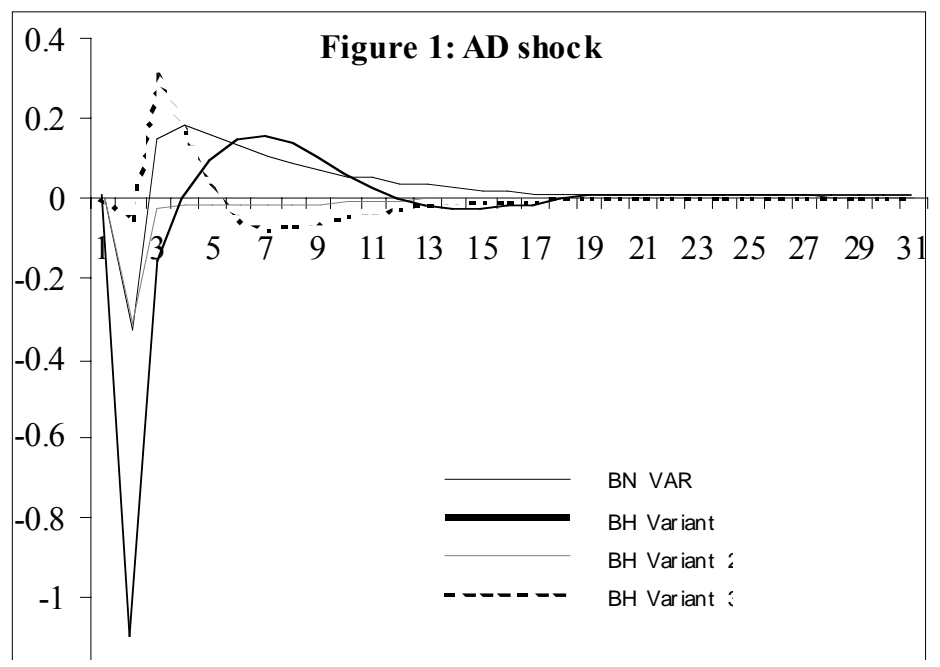


The impulse response of inflation for the various models exhibits a similar pattern: inflation falls on impact and then converges to target, with little or no overshooting. Under an optimal rule the interest rate responds strongly and in a countercyclical fashion to output, so that the inflation response following an $\mathrm{AD}$ shock is actually negative. For example, in the VAR model, current output enters the optimal interest rate rule with a coefficient of 8.82 . However, as one would expect, each model behaves somewhat differently under the optimal rule, and the time at which inflation settles back on target after the AD shock varies across models.

In the case of the VAR, because of its highly persistent estimated dynamics, inflation moves back to target sluggishly. Table 3 suggests that it takes ten quarters for inflation to go back to its initial value using the \pm 0.05 percent absolute criterion. The backward-looking IS variant of the BH model (BH Variant 1) shows a more vigorous response of the interest rate to a sudden temporary rise in output $(1.3038 \%$ versus -0.3303 in the VAR), eliciting a much deeper initial fall of inflation on impact $(-1.093 \%)$. As with the VAR, in this model, stickiness from the contracting specification, together with a lack of forward-looking behaviour in aggregate demand, implies considerable persistence in inflation. This means that again, it takes ten quarters for inflation to be stabilised under an optimal rule (Table 4).

In contrast, in the second variant of the $\mathrm{BH}$ model (BH with forward-looking IS) the expectational dynamics of the IS curve reduce the length of the transmission lag and thus, speed up the stabilisation property of the optimal policy rule. Here, the length of the optimal policy horizon under the \pm 0.05 percent absolute criterion is a fifth of the policy horizon that is optimal for the VAR and BH Variant 1 models, suggesting that it is optimal to carry out the disinflation within two quarters of the shock (Table 5). Finally, when we allow aggregate supply to be fully forward-looking (a là 
quarters to bring inflation on target under the $k_{A 2}$ criterion and our baseline specification of weights. Counterintuitively, this is the same time it takes policy to bring inflation back on target in the BH model with a backward-looking IS function and mixed backward/forward-looking aggregate supply. A priori we would expect a shorter lag because of the far smaller degree of inherent inflation persistence in the Taylor specification of aggregate supply. The reason why $k$ is large is that, as the Figure shows, although inflation returns to target quite rapidly (four quarters) it then overshoots and undershoots the target for several periods, leading to a large value of $k$.

Aggregate supply shock

Figure 2 shows the impulse responses of inflation for the four models, in the wake of a temporary $1 \%$ shock to aggregate supply.

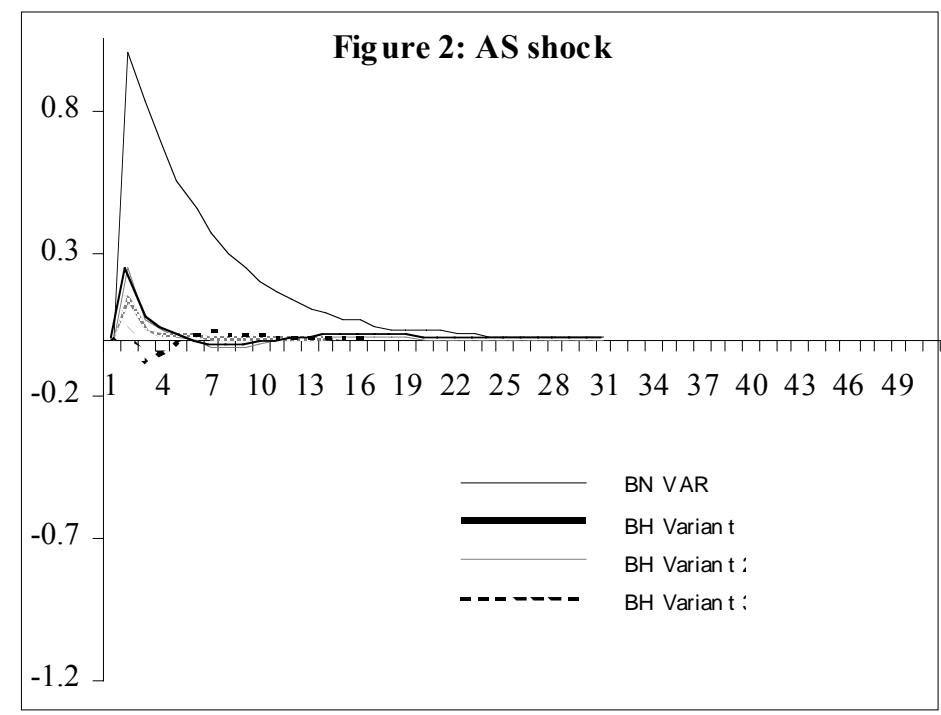

For this aggregate supply disturbance, the unrestricted VAR displays quite smooth dynamics for inflation. With monetary policy unable to affect inflation in the period of the shock, the $1 \%$ supply shock raises inflation by a full one percent in the first period. Then it takes sixteen quarters for this shock to be corrected completely 
$\left(k_{A 2}=k_{R}=16\right)$. In the case of $\mathrm{BH}$ Variant 1 , the response of inflation under the AS shock is substantially smaller in the initial period because the interest rate can contemporaneously affect inflation, in contrast to the VAR where the effect is lagged by construction. So under our baseline weight combination, the optimal policy cuts the effect on inflation of the supply innovation from (what would have been) $1 \%$ to $0.23 \%$. However, because of the inherent persistence of inflation in the aggregate supply specification employed here, and because of the lack of forward-looking behaviour in the IS function, it takes three quarters to be dragged back to target under the $k_{A 2}$ criterion, with undershooting then preventing inflation from settling back at target for eleven quarters under the $k_{R}$ criterion.

In the case of BH Variant 2, the presence of expected future output in the aggregate demand equation enables policymakers under the optimal rule to stabilise inflation within a \pm 0.05 percent zone around target within just two quarters. Inflation is kept closer to the target in two ways. First, the initial impact of inflation in response to the shock is smaller vis-à-vis the preceding backward-looking models (i.e. the VAR and the BH Variant 1) - optimal policy, by affecting output and expected inflation appropriately, succeeds in offsetting much of the effect on inflation of the supply shock. Secondly, forward-looking behaviour reduces the extent to which inflation has protracted departures from target following a shock (so that, in BH Variant 1, inflation is brought back to target in a shorter time, from three to two quarters using the absolute criterion, $k_{A 2}$, and from eleven to nine using the relative criterion, $k_{R}$ ).

Under Taylor contracting (BH Variant 3) the positive impact effect on inflation of the AS shock is almost entirely removed by the optimal policy response. In effect, policy produces a negative movement in $\mathrm{E}_{t} \pi_{t+1}$ of almost equal and opposite magnitude to that of the supply shock, essentially cancelling the shock's effect on the right-hand-side of equation (1.4). Following this subdued initial response of inflation, there is a minor undershooting followed by an even smaller overshooting. Under the 
\pm 0.05 percent absolute criterion this implies a $k_{A 2}{ }^{*}$ of three quarters, whereas under the relative criterion, the lingering movement of inflation away from target implies a much greater $k_{R}^{*}$ (18 quarters).

\section{Exchange rate shock}

Figure 3 shows the impulse responses of inflation for the four models described in Section 1 , in the wake of a temporary $1 \%$ shock to the exchange rate.

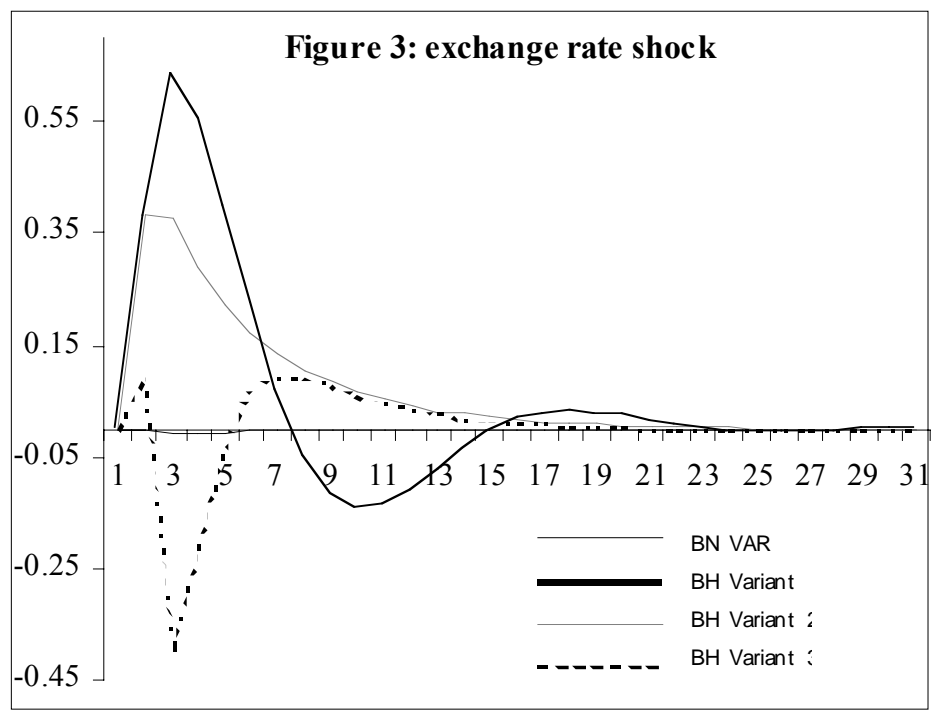

The estimated VAR contains an exchange rate equation that is essentially detached from the rest of the model, in the sense that there is virtually no feedback to other variables from the exchange rate. ${ }^{7}$ Consequently under the optimal policy the exchange rate enters with a very small coefficient $(-0.0301)$. This determines the basically flat response of inflation to such a shock (in Figure 3, the VAR's inflation response is indistinguishable from the $\mathrm{x}$-axis). As a result, under the \pm 0.05 percent absolute criterion, inflation is always on target, i.e., $k_{A 2}{ }^{*}=0$; and under the relative criterion, there are economically negligible effects on inflation at all lags, but $k_{R}{ }^{*}=18$ 
because $5 \%$ of the negligible impact effect on inflation remains in the system for a lengthy time.

The appearance of the exchange rate in both the IS and the AS functions with sizeable coefficients implies an important effect of the exchange rate on other variables in all variants of the $\mathrm{BH}$ model compared to the VAR. In the case of BH Variant 1, under optimal policy, a $1 \%$ shock to the exchange rate exerts its effects with lags on output and inflation and has its peak effect on inflation with a one quarter lag, as the indirect output gap channel becomes effective; it then stays at this peak for another period, after which the optimal policy brings it down around target after one year. But a sequence of undershoots then extends the deviations from target so, by the \pm 0.05 percent absolute criterion, $k_{A 2}{ }^{*}$ is large at thirteen. The relative criterion implies an even longer optimal policy horizon, with $k_{R}{ }^{*}=20$.

When the IS curve is forward-looking (BH Variant 2), the initial effect of the exchange rate on inflation is smaller than in the backward-looking case, at about 0.4. This peak also occurs at no lag, in contrast with the backward-looking case; in part this is because policy can now affect output contemporaneously, and output has a contemporaneous effect on inflation. Although here optimal policy does not cause the undershooting that it produces in the backward-looking case, it still takes a long time to bring inflation on target. The $k_{A 2}{ }^{*}$ is eleven, in contrast to the $k_{A 2}{ }^{*}$ of thirteen in $\mathrm{BH}$ Variant 1. Correspondingly, $k_{R}{ }^{*}$ is fifteen, where it is was twenty in BH Variant 1.

Taylor contracting (BH Variant 3) allows the initial response of inflation to an exchange rate shock to be much smaller under the optimal policy than in the other two models. But there is then a serious undershooting followed by a prolonged overshooting, producing long optimal horizons of $k_{A 2} *=10$ and $k_{R} *=18$.

\section{2.a.1 Results for different sets of weights}


In addition to the baseline results discussed above, Tables 3 to 6 present results for $k_{A 2}{ }^{*}$ and $k_{R}{ }^{*}$ when the weights in the loss function $(1.1)$ are $\left(\lambda=1.0, \lambda_{y}=0\right)$ and $(\lambda=0.5, \lambda y=1.0)$, respectively.

For the VAR, increasing the weight on inflation shortens the optimal horizon, as inflation control gets tighter, but the optimal horizon remains above ten for the aggregate demand and the aggregate supply shocks for both the ' $A 2$ ' and ' $R$ ' definitions of $k^{*}$. If we instead increase the relative weight on output, the second absolute- and relative-criterion OPHs under aggregate supply and exchange rate shocks are unchanged; for an aggregate demand shock, however, we find that $k_{A 2}{ }^{*}$ and $k_{R}{ }^{*}$ do change, and go in opposite directions (with $k_{A 2}{ }^{*}$ rising to twenty quarters and $k_{R}{ }^{*}$ falling to sixteen; see Table 3).

In the case of the $\mathrm{BH}$ model Variant 1, placing a zero weight on output actually produces non-convergent results for most shocks: inflation oscillates around target indefinitely and there are signs of instrument instability (which leads to infinite OPHs under both $k_{A 2}$ and $k_{R}$ criteria). ${ }^{8}$ This is because in this backward-looking model, a zero weight on output leads to large swings in the interest rate instrument. When output is assigned a greater weight than inflation, $k_{A 2} *$ is largely unchanged, whereas, somewhat surprisingly, $k_{R}^{*}$ falls for all types of shocks (see Table 4).

In a model with more forward-looking elements than the VAR or BH Variant 1, anticipation of future policy action by private agents leads to a smoother response of output and to a tighter control of inflation. This is evident in Tables 5 and 6 , which feature the variants of the $\mathrm{BH}$ model that allow for, respectively, forward-looking aggregate demand and fully forward-looking price-setting. Under either of these modifications, setting the weights to $\left(\lambda=1.0, \lambda_{y}=0\right)$ leads to $k_{A 2} *$ falling to zero for 
all shocks, while it makes $k_{R}{ }^{*}$ sharply lower, in the seven to eleven quarters range. Putting higher weight on output than on inflation uniformly lengthens the values of $k_{A 2}{ }^{*}$ and $k_{R}{ }^{*}$ for all shocks relative to the baseline case. 


\section{Optimal feedback horizons (OFHs)}

The previous section searched for optimal policy horizons for a suite of models assuming that the policymakers followed a complex optimal rule — allowed to be a function of the entire state vector. Suppose, instead, that the inflation-targeting policymakers operate via a simple, explicit policy rule that involves moving the interest rate (the operating variable) to correct deviations of expected inflation (the intermediate variable) from its target value, that is via a rule like:

$R_{t}=\psi_{p}\left(\mathrm{E}_{t-1} \pi_{t+k}-\pi^{T A R}\right)$

where $R_{t}$ is the short-term nominal interest rate, $\pi_{t}$ is inflation, $\pi^{T A R}$ is the inflation target and $\mathrm{E}_{t-1}$ is the expectation operator at time $t-1$, so that expected inflation deviations from target become an explicit feedback variable.

Rules like (3.1) have been investigated by Coletti et al (1996), de Brouwer and O’Regan (1997), Batini and Haldane (1999), and Black, Macklem and Rose (1998).

These rules are similar to standard simple current feedback instrument rules inasmuch as they feed back only on a subset of variables rather than on the whole state vector like complex optimal rules. But they differ from such rules because they react to deviations of expected (rather than actual) inflation from target. By suitable choice of the feedback horizon, these rules can be designed so as to embody, automatically, monetary transmission lags. In particular, the feedback variable in the rule can be chosen so that it is subject to potential influence by the monetary authorities. Also, by reacting to a variable, expected inflation, that is itself a function of the wider state vector, these rules should be closer to the optimal one.

When inflation targeting is implemented through rules like (3.1), the best $k$-periodahead forecast of inflation to use as an intermediate target is the one from which it is most efficient to correct deviations. In mathematical terms, the optimal horizon for 
inflation targeting will be the $k$ associated with (3.1) that minimises the costs of inflation stabilisation for a given social welfare function: we define this horizon as the optimal feedback horizon (OFH). Two things are worth noting here: first, we consider 'optimal' only the feedback horizon that minimises the costs of inflation stabilisation under rule (3.1) when the rule's coefficients are optimally chosen. This implies that the choice of the optimal $k$ is conditioned on $\psi_{p}$ (and $\rho_{R}$ when a term for interest rate smoothing is included) being optimal. It follows that we call 'OFHs' only those horizons that are optimal when (3.1) is a simple optimal rule, while we call 'FHs' all the other horizons associated with simple non-optimal rules. Second, here, in contrast with the previous section, the optimal horizon is not a concept that can be bracketed by a range. Instead, by construction, it can only be a discrete point (i.e. the best $k$ at which to form a forecast on inflation on which to feed back). So from now on, OFHs are associated with only one $k$, and not with a series of them (from various criteria, say).

In this section we derive optimal feedback horizons for each of the models described in Section 1 (BN VAR, and BH Variants 1, 2 and 3). For that purpose, we generalise rule (3.1) in two respects. First, we modify (3.1) to include a coefficient on the lag of the nominal interest rate (an interest rate smoothing term). There are several reasons why the monetary authorities may want to smooth interest rates. First, smoothing may prevent placing 'undue stress' on the financial markets (Goodhart (1996)). Second, it minimises the risk of policy reversals which, some have argued, may endanger credibility (Meulendyke (1990)). Third, having a uniform interest rate path may communicate policy intentions most clearly and credibly to markets (Goodfriend (1991)). ${ }^{1}$ Fourth, smoothing may be preferable in the face of multiplicative and/or model uncertainty (Brainard (1967), Sack (1997), Martin (1998)); finally, diluting the interest rate response over time may facilitate policy setting in the absence of unambiguous current evidence of significant deviations of actual from target outcomes

\footnotetext{
${ }^{1}$ On a related point, Rotemberg and Woodford (1998) find that a high degree of interest rate smoothing re-inforces the effectiveness of an anti-inflationary monetary policy due to the effects on agents'
} 
(Goodhart (1996)). Inclusion of a smoothing term in equation (3.1) gives (3.2) below, where the degree of interest rate smoothing is governed by the parameter $\rho_{R} . \rho_{R}$ is, in turn, defined over the interval $[0,1)$, with $\rho_{R}=0$ signifying 'no smoothing'.

$R_{t}=\rho_{R} R_{t-1}+\psi_{p}\left(\mathrm{E}_{t-1} \pi_{t+k}-\pi^{T A R}\right)$

Secondly, we allow the nominal rate to correct the average deviation from target of expected inflation over a number of periods in time — rather than just one deviation from target of inflation expected at some specific future horizon. ${ }^{2}$ There are both practical and conceptual grounds for feeding back on an average of inflation expectations. A practical ground is that the Bank of England (or, more generally, inflation-targeting central banks) targets a yearly inflation measure. Therefore, responding to a three- or four-quarter average of deviations of expected inflation from target may approximate more closely what policymakers do in practice. A conceptual ground is that if the empirical response of inflation to changes in the interest rate is hump-shaped - implying a peak response at some intermediate quarter, but also some less-than-peak responses in adjacent quarters — reacting to an average of expectations may be more effective at stabilising prices because it exploits more fully the impact of the marginal inflation response. Equation (3.3) below illustrates algebraically such an average feedback rule: in period $t$ policy reacts to a weighted sum (with weights $\gamma_{k}$ ) of deviations of inflation forecasts - measured at various horizons - from target, where all forecasts are conditional on the same information at time $t$. As in (3.1) and (3.2), the coefficient on this average of deviations is $\psi_{p}$; and it is possible to smooth interest rates by suitable choice of $\rho_{R}$. Thus in this case the feedback horizon is in fact an average feedback horizon (AFH) and the optimal feedback horizon is an optimal average feedback horizon (OAFH). We will make use of this terminology in what follows.

$$
R_{t}=\rho_{R} R_{t-1}+\psi_{p} \sum^{M} \gamma_{k}\left(\mathrm{E}_{t-1} \pi_{t+k}-\pi^{T A R}\right)
$$


To identify the OFH or the OAFH, we simulate the models stochastically, closing them either with rule (3.2) or (3.3). ${ }^{3}$ The OFH (OAFH) is the $k$ (group of $k \mathrm{~s}$ ) that minimises the average loss function across each set of simulations.

However, since the derivation of the optimal $k$ (group of $k \mathrm{~s}$ ) is conditioned on the choice of the other policy parameters in the rules (namely $\psi_{p}, \gamma_{k}, \rho_{R}$ and $M$ ), we search for $k \mathrm{~s}$ (group of $k \mathrm{~s}$ ) under various assumptions about these parameters. In particular, we derive the optimal $k$ (optimal group of $k \mathrm{~s}$ ) for rule (3.2) when:

(i) $\rho_{R}=0$ (no smoothing) and $\psi_{p}$ is arbitrary (non-optimised);

(ii) $\rho_{R}$ is optimised (optimal smoothing) $\left(1>\rho_{\mathrm{R}} * \geq 0\right)$ and $\psi_{p}$ is arbitrary (nonoptimised);

(iii) $\rho_{R}=0$ (no smoothing) and $\psi_{p}$ is optimised $\left(\psi_{p}^{*}\right)$;

(iv) $\rho_{R}$ is optimised (optimal smoothing) $\left(1>\rho_{\mathrm{R}} * \geq 0\right)$ and $\psi_{p}$ is optimised $\left(\psi_{p} *\right)$.

Throughout, the contemplated values of $k$ are $0,1, \ldots, 10$.

For rule (3.3), the set of average feedback horizons (we denote the moving average of $k$ by ' $\Sigma k_{i}$ ' from here on) includes three inter-period averages only, i.e., for $\tau=1$, $\Sigma k_{1}=(t+1, \ldots, t+4) ;$ for $\tau=4, \Sigma k_{2}=(t+4, \ldots, t+7) ;$ and finally, for $\tau=7$, $\Sigma k_{3}=(t+7, \ldots, t+10)$, and $M$ is set equal to $(\tau+3)$ throughout. ${ }^{4}$ We experiment with two kinds of weights on the components of the moving averages $\left(\gamma_{k}\right)$ : first a set of equal weights $(1 / M)$; second, weights (with their sum normalised to one) based on the

\footnotetext{
${ }^{3}$ Details on the simulation techniques are provided in the Technical Appendix. For the BN VAR, as in Section 2, we simulate the system of equations (1.2) replacing the estimated policy rule with the hypothetical rule (3.2).
} 
relative importance of each lag in a distributed lag regression of inflation on interest rates (see the Technical Appendix for further details). In the tables below, we refer to these weighting schemes as 'W1' and ' $\mathrm{W} 2$ ' respectively.

We search for optimal $k$ s when $\psi_{p}$ is arbitrarily fixed - (i) in the list above because some of our models display little trade-off between inflation and output variability and hardly any tendency for instrument instability. The latter is basically absent because these models have a simple lag structure with roots well away from the region of dynamic instability. ${ }^{5}$ This implies that the loss function (1.1) in Section 1 above can sometimes be reduced just by increasing $\psi_{p}$ almost without limit. ${ }^{6}$ If this is the case, it may be useful to identify the optimal value of $k$ for lower - fixed values of $\psi_{p}$.

The section is organised as follows. In part (3.a) we derive numerically FHs, OFHs and AFHs for the various models. In a subsection (3.b) we study how the optimal $k \mathrm{~s}$ (the OFHs) relate to the corresponding (same model/loss function/ shock) OPHs of Section 2.

\section{3.a Numerical results}

We start the analysis of optimal feedback horizons by identifying the $k s$ associated with the simple feedback rule (3.3) that minimise the costs of inflation stabilisation for a given social welfare function and given set of policy choice parameters. Tables 7-10 summarise the results on feedback horizons (FHs) when the models are closed by rule (3.2) (i.e. by the simple feedback rule with a term for interest rate smoothing).

Table 7 lists OFHs when $\rho_{R}=0$ (no smoothing) and $\psi_{p}$ is arbitrary (non-optimised).

\footnotetext{
${ }^{5}$ This is not true for the BN VAR model. However, in that case, the estimated effects of the interest rate on the other variables is so small that the model's dynamics can be stable even with very large feedback coefficients in the rule.

${ }^{6}$ This is made evident by some outlandishly large 'optimal' values of $\psi_{p}$ that appear in Tables 9 and
} 
Table 8 shows OFHs when $\rho_{R}$ is optimised (optimal smoothing) over the grid $[0,0.1, \ldots, 0.9]$ and $\psi_{p}$ is arbitrary (non-optimised). For both tables, the arbitrary values used for $\psi_{p}$ are 1.5 and 3.0, which are close to values normally used in the literature for these kinds of rules. ${ }^{7}$ Loss function values are computed using equation (1.1), with weights $\lambda \pi=\lambda y=0.5$, throughout.

\begin{tabular}{|c|c|c|c|}
\hline \multicolumn{1}{|c|}{ Table 7: } & FHs - Arbitrary $\psi_{p}, \rho_{R}=0$ (no smoothing ) \\
\hline Model & $\psi_{p}$ & $k(F H s)$ & Loss \\
\hline BN VAR & 1.5 & 8 & 97.27 \\
\hline BH Variant 1 & 1.5 & 1 & 137.88 \\
\hline BH Variant 2 & 1.5 & 4 & 55.40 \\
\hline BH Variant 3 & 1.5 & 4 & 7.37 \\
\hline & & & \\
\hline BN VAR & 3.0 & 8 & 84.04 \\
\hline BH Variant 1 & 3.0 & 2 & 107.47 \\
\hline BH Variant 2 & 3.0 & 4 & 52.78 \\
\hline BH Variant 3 & 3.0 & 5 & 74.45 \\
\hline
\end{tabular}

\begin{tabular}{|c|c|c|c|c|}
\hline \multicolumn{4}{|c|}{ Table 8: FHs - Arbitrary $\psi_{p}, 1.0>\rho_{R} \geq 0$ (smoothing) } \\
\hline \multirow{2}{*}{ Model } & $\psi_{p}$ & $\rho_{R}{ }^{*}$ & $k$ (FHs $)$ & Loss \\
\hline BN VAR & 1.5 & 0.7 & 9 & 86.88 \\
\hline & 3.0 & 0.9 & 10 & 79.48 \\
\hline BH Variant 1 & 1.5 & 0.3 & 2 & 115.56 \\
\hline & 3.0 & 0.0 & 2 & 107.47 \\
\hline
\end{tabular}




\begin{tabular}{|c|c|c|c|c|}
\hline BH Variant 2 & 1.5 & 0.1 & 4 & 55.49 \\
\hline & 3.0 & 0.0 & 4 & 52.78 \\
\hline BH Variant 3 & 1.5 & 0.2 & 5 & 76.12 \\
\hline & 3.0 & 0.1 & 5 & 73.81 \\
\hline
\end{tabular}


Several key features are apparent from Table 7:

(i) For all models, the welfare-maximising value of $k$ is greater than zero. This is in line with the argument of, inter alia, Batini and Haldane (1999) that there is merit in the policymaker being forward-looking (i.e. $k>0$ in rule (3.1)) when sluggishness in private agents' behaviour delays the response of inflation to changes in monetary policy.

(ii) The VAR tends to support a higher value of $k, 8$, than the other models. This reflects the nature of the VAR estimates. As we discussed in Section 1, the BN VAR (like many other VARs) implies that interest rates have a correctly signed effect on inflation only after several periods, as the output gap channel becomes effective. Therefore, rule (3.3) performs poorly for low values of $k$. As we also noted, the VAR estimates imply a sluggish inflation process: this also supports a higher value of $k$, since policy can more easily affect medium-run inflation than inflation in the near future. ${ }^{8}$

(iii) The most backward-looking models — the BN VAR and BH Variant 1 - deliver the poorest loss function values. This reflects the fact that, in these models, expectations of policy behaviour play a minor role - or no role in the BN VAR — in private agents' pricing and spending decisions. The more forward-looking a model, the more the policymaker can create stability by maintaining an antiinflationary policy, relying largely on the threat of action on interest rates if aggregate demand or inflation become inconsistent with policy goals. If agents factor such a reaction function into their decisions, then expectations of output relative to capacity will be relatively stable. In forward-looking models, then, the expectation of antiinflationary monetary policy itself tends to create a stable path for output and

\footnotetext{
${ }^{8}$ One means by which policy may have a rapid effect on inflation is through the exchange rate channel. But according to our VAR estimates this channel has virtually no role, in contrast to the BH model
} 
inflation, leading to low values of the loss function (1.1). Clearly, this cannot occur in a backward-looking model, hence the greater costs of inflation stabilisation.

(iv) Although it is the most backward-looking of the BH variants, for nonoptimised $\psi_{p}$, Variant 1 appears to favour a lower FH value of $k$ than the other two variants. On the face of it, this is surprising because in a very backward-looking model, inflation has little scope to "jump", increasing the attractiveness of feeding back on inflation far ahead. However, this should be put into perspective by the fact that the more forward-looking models (Variants 2 and 3) tend to produce uniformly lower loss function values than BH Variant 1, regardless of $k$. For example, for $\psi_{p}=3.0, k=4$ delivers a smaller loss function value than $k=1$ for $\mathrm{BH}$ Variant 2 ; nevertheless, $k=1$ would give a lower value of the loss function under $\mathrm{BH}$ Variant 2 than under BH Variant 1.

(v) Finally, raising the response parameter from 1.5 to 3.0 uniformly reduces the loss function value in all models. Thus, instrument instability is not a serious problem over our chosen parameter range.

Turning to Table 8 , the models generally indicate little gain from interest rate smoothing relative to the no-smoothing case. The main exception is the VAR. Here, a high degree of smoothing ( $\rho_{R}=0.7$ or 0.9 ) is favoured over the no-smoothing case, and $k$ increases from 8 (no smoothing) to $9\left(\rho_{R}=0.7\right)$ or $10\left(\rho_{R}=0.9\right)$. The better results from smoothing probably come from two main sources: (i) greater short-run output stability due to the smoother interest rate profile; (ii) greater longer-run inflation stability arising from the implied increase in the long-run inflation response from $\psi_{p}$ to $\psi_{p} /\left(1-\rho_{R}\right)$. Indeed, it is surprising that these gains from smoothing do not appear important in the BH model variants, all of which are forward-looking in some respect; it has been argued that interest rate smoothing can generate macroeconomic benefits when there are many forward-looking elements in a model (Rotemberg and Woodford [1999]). 
Table 9 shows forecast horizons when again $\rho_{R}=0$ (no smoothing) but now $\psi_{p}$ is derived from a (grid-search based) optimisation exercise; and Table 10 shows OFHs when both $\rho_{R}$ and $\psi_{p}$ are optimised.

As we foreshadowed, the 'optimal' values of $\psi_{p}$ turn out to be very large in some cases - especially in the VAR, whose backward-looking, data-based nature might instead have led us to expect instrument instability when policy becomes too aggressive. Evaluating the VAR model's properties where the feedback parameters take such large values may take the model into ranges where it ceases to be a useful approximation for policy analysis. For this reason, we would emphasise the results on that model from the more constrained experiments of Tables 7 and 8, and simply note that the optimal $k$ in Table 9 and 10 for the VAR is 9 or 10 , corroborating the high optimal values of $k$ found in Tables 7 and 8 for the VAR model.

Table 10 indicates that in all variants of the $\mathrm{BH}$ model, the loss function is minimised by policies that avoid explicit interest rate smoothing. The optimal feedback parameter is around 3 for both $\mathrm{BH}$ Variants 1 and 2, with the optimal $k$ being 2 and 4 quarters respectively; for BH Variant 3, by contrast, the optimal policy has a shorter $k$ (namely, one) than the other BH Variants (overturning the results of Table 7 and 8) and a larger feedback parameter $\left(\psi_{p}^{*}=7.5\right)$. This model features the most forwardlooking price setting behaviour of all the models under consideration, which makes inflation 'jumpier' and more sensitive to expectations of monetary policy. Such an aggregate supply specification evidently makes it more attractive to target near-future inflation, and to respond aggressively to expected deviations from target. 


\begin{tabular}{|c|c|c|c|}
\hline \multicolumn{2}{|c|}{ Table 9: } & FHs - Optimised $\psi_{p}, \rho_{R}=0$ (no smoothing) \\
\hline Model & $\psi_{p}{ }^{*}$ & $k(F H s)$ & Loss \\
\hline BN VAR & 27.30 & 10 & 59.07 \\
\hline & & & \\
BH Variant 1 & 3.35 & 2 & 104.10 \\
\hline & & & 53.21 \\
\hline BH Variant 2 & 3.00 & 4 & 67.81 \\
\hline
\end{tabular}

\begin{tabular}{|c|c|c|c|c|}
\hline \multicolumn{2}{|c|}{ Table 10: } & OFHs - Optimised $\psi_{p}$, optimised $\rho_{R}\left[1>\rho_{R} \geq 0\right.$ (smoothing) $]$ \\
\hline Model & $\rho_{R}{ }^{*}$ & $\psi_{p}{ }^{*}$ & $k^{*}($ OFHs $)$ & Loss \\
\hline BN VAR & 0.1 & 40.85 & 9 & 58.02 \\
\hline & & & & \\
BH Variant 1 & 0.0 & 3.35 & 2 & 104.10 \\
\hline & & & & 53.21 \\
\hline BH Variant 2 & 0.0 & 3.00 & 1 & 67.81 \\
\hline
\end{tabular}

Tables 11 and 12 present average feedback horizons (AFHs) based on weighting scheme W1 (equal weights), while Tables 13 and 14 present AFHs based on weighting scheme W2 (regression-based weights). A comparison of Tables 8 and 14 indicates that, for $\mathrm{BH}$ Variant 1 , feeding back on $\Sigma k_{2}$ (i.e., a moving average of $k$ s from 4 to 8 weighted according to scheme W2) is better than feeding back on any single $k$. This suggests that using averages of lags in a policy rule may deliver some benefits. However, such benefits are not evident in the remaining results in Tables 11-14; averaging under either weighting scheme fails to produce superior outcomes than the simpler, non-averaged rules in Tables 7 and 8 . But this may be a consequence of the fact that in Tables 11 and 12 the rule coefficients are not both optimised. One 
possible extension would be to investigate OAFHs (and perhaps AFHs and OAFHs with new weighting schemes). 


\begin{tabular}{|c|c|c|c|}
\hline \multicolumn{4}{|c|}{ Table 11: AFHs - Average Rules (W1) } \\
Arbitrary $\psi_{p}, \rho_{R}=0$ (no smoothing) \\
\hline Model & $\psi_{p}$ & $\Sigma k(A F H s)$ & Loss \\
\hline BH Variant 1 & 1.5 & $\Sigma k_{1}$ & 172.56 \\
\hline BH Variant 2 & 1.5 & $\Sigma k_{2}$ & 59.27 \\
\hline BH Variant 3 & 1.5 & $\Sigma k_{2}$ & 78.11 \\
\hline BH Variant 1 & 3.0 & $\Sigma k_{2}$ & 146.05 \\
\hline BH Variant 2 & 3.0 & $\Sigma k_{2}$ & 56.78 \\
\hline BH Variant 3 & 3.0 & $\Sigma k_{2}$ & 76.62 \\
\hline
\end{tabular}

\begin{tabular}{|c|c|c|c|c|}
\hline \multicolumn{5}{|c|}{ Table 12: AFHs - Average rules (W1) } \\
Arbitrary $\psi_{p}, 1.0>\rho_{R} \geq 0$ (smoothing) \\
\hline \multirow{2}{*}{ Model } & $\psi_{p}$ & $\rho_{R}{ }^{*}$ & $\Sigma k$ (AFHs) & Loss \\
\hline BH Variant 1 & 1.5 & 0.5 & $\Sigma k_{2}$ & 116.32 \\
\hline & 3.0 & 0.1 & $\Sigma k_{2}$ & 105.68 \\
\hline BH Variant 2 & 1.5 & 0.3 & $\Sigma k_{2}$ & 59.06 \\
\hline & 3.0 & 0.0 & $\Sigma k_{2}$ & 56.78 \\
\hline BH Variant 3 & 1.5 & 0.2 & $\Sigma k_{2}$ & 77.34 \\
\hline & 3.0 & 0.2 & $\Sigma k_{2}$ & 76.00 \\
\hline
\end{tabular}




\begin{tabular}{|l|c|c|c|}
\hline \multicolumn{4}{|c|}{$\begin{array}{c}\text { Table 13: AFHs - Average Rules (W2) } \\
\text { Arbitrary } \psi_{p}, \rho_{R}=0 \text { (no smoothing) }\end{array}$} \\
\hline Model & $\psi_{p}$ & $\Sigma k(A F H s)$ & Loss \\
\hline BH Variant 1 & 1.5 & $\Sigma k_{1}$ & 177.63 \\
\hline BH Variant 2 & 1.5 & $\Sigma k_{2}$ & 59.69 \\
\hline BH Variant 3 & 1.5 & $\Sigma k_{2}$ & 78.58 \\
\hline BH Variant 1 & 3.0 & $\Sigma k_{1}$ & 109.06 \\
\hline BH Variant 2 & 3.0 & $\Sigma k_{2}$ & 56.59 \\
\hline BH Variant 3 & 3.0 & $\Sigma k_{2}$ & 76.20 \\
\hline
\end{tabular}

\begin{tabular}{|c|c|c|c|c|}
\hline \multicolumn{5}{|c|}{ Table 14: AFHs - Average rules (W2) } \\
Arbitrary $\psi_{p}, 1.0>\rho_{R} \geq 0$ (smoothing) \\
\hline \multirow{2}{*}{ Model } & $\psi_{p}$ & $\rho_{R}{ }^{*}$ & $\Sigma k(A F H s)$ & Loss \\
\hline BH Variant 1 & 1.5 & 0.6 & $\Sigma k_{1}$ & 114.85 \\
\hline & 3.0 & 0.2 & $\Sigma k_{2}$ & 107.09 \\
\hline BH Variant 2 & 1.5 & 0.2 & $\Sigma k_{2}$ & 57.57 \\
\hline & 3.0 & 0.1 & $\Sigma k_{2}$ & 55.62 \\
\hline BH Variant 3 & 1.5 & 0.2 & $\Sigma k_{2}$ & 77.53 \\
\hline & 3.0 & 0.3 & $\Sigma k_{2}$ & 75.93 \\
\hline
\end{tabular}




\section{3.b A comparison of OFHs and $\mathrm{PHs} / \mathrm{OPHs}$}

So far we have identified two kinds of horizon: a 'policy horizon', i.e. the time at which inflation settles on target, and a 'feedback horizon', i.e. the date of the inflation forecast on which policymakers feed back. We showed that both these horizons can be chosen to be optimal. For the policy horizon, this implies determining the number of periods from now at which inflation is back on target under an optimal rule, after a shock today - i.e., the $k$ consistent with restoring inflation to target at minimal social cost: the 'optimal policy horizon' (OPH). Although in the context of these optimal control experiments, the horizon is not really a choice variable; rather it drops out of applying the optimal rule. The point is that the resulting 'horizon' may be instructive to real world policymakers trying to get inflation in line with target.

The feedback horizon approach involves identifying the date in the future on which it is least costly to feed back using a simple policy rule with optimised parameters: the 'optimal feedback horizon' (OFH). When, instead, policymakers feed back on averages of expected inflation deviations from target, the feedback horizon is in fact an 'average feedback horizon'; that becomes an 'optimal average feedback horizon' (OAFH) when it is chosen optimally.

In this subsection we address two questions: (i) when does inflation return to target under rules that employ optimal feedback horizons (OFHs), i.e. what (non-optimal) policy horizons are implied by these rules?; and (ii) how do these (non-optimal) policy horizons relate to their corresponding (i.e. same model, loss function, and shock) optimal policy horizons of Section 2 ?

For this purpose, we compute policy horizons under both the absolute and relative criteria of Section 2 when the model is closed by parametrisations of the simple rules (3.2) and (3.3) that are associated with the optimal feedback or optimal average 
when the models are closed with rule (3.2) and optimised parameters from Table 10 for each shock. As in Section 2, this is done by deriving impulse response functions for an aggregate demand, aggregate supply and exchange rate shock in our four models, and identifying the dates $(\mathrm{ks})$ at which inflation enters and remains in the assigned target zone under each criterion. On the basis of these impulse responses, we can then compute policy horizons for each model under the various shocks. In line with Section 2, we consider policy horizons that are defined over target zones rather than point targets; i.e., we think of a policy horizon as the time at which inflation returns to, and remains thereafter inside, a specified band around the target, rather than as the time at which inflation settles back exactly on target after an inflationary shock. Also, as in Section 2, we use three alternative criteria to define a policy horizon: two absolute criteria - that is, the number of periods, $k$, from now when inflation has returned permanently to within a target zone of $\pm 1 \mathrm{pp}$ (the ' $k_{A 1}$ ' criterion) and \pm $0.05 \mathrm{pp}$ (the ' $k_{A 2}$ ' criterion) around target after a shock today; and a relative criterion - that is the number of periods $(k)$ from now at which $95 \%$ of the initial effect of the shock on inflation has been permanently eroded (the ' $k_{R}$ ' criterion). Note, however, that since the policy horizons derived here are non-optimal (because they are not the times at which inflation is on target under a fully optimal rule, but rather, when inflation is on target under simple rules with optimised coefficients) their labels are not starred.

Figures 4 - 6 below show the models' impulse responses for each shock. 


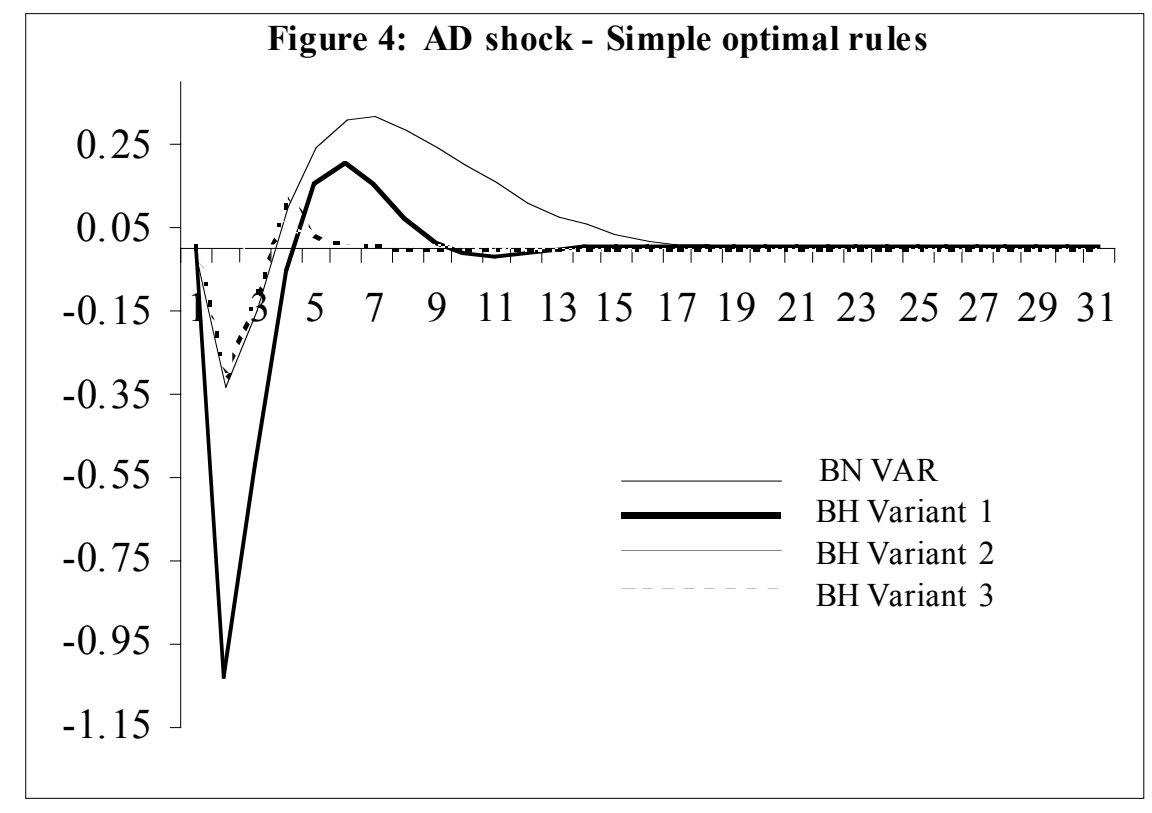

Figure 4 illustrates the impulse responses of inflation for the four models, in the face of a temporary, $1 \%$ positive shock to aggregate demand. The responses under the simple optimal rule (3.2) are similar to the ones obtained under the fully optimal rule in Section 2 (see Figure 1). For most models (BN VAR, BH Variants 1 and 3), after an $\mathrm{AD}$ shock the interest rate responds aggressively to offset the inflationary rise in demand, so that inflation actually falls on impact. Inflation falls on impact for the $\mathrm{BH}$ Variant 2 model too, although in this case the interest rate initially falls rather than rises after the shock. Again, as under the fully optimal rule, the simple optimal rule produces a sluggish return of inflation to target after the shock in the more backwardlooking models (the BN VAR and BH Variant 1), which have more persistent inflation dynamics. This produces rather long policy horizons for these models ( $k_{A 2}$ is 14 for the BN VAR and 8 for BH Variant $1 ; k_{R}$ is 16 for the BN VAR, $k_{A 2}$ and $k_{R}$ are the same (eight) for $\mathrm{BH}$ Variant 1). 


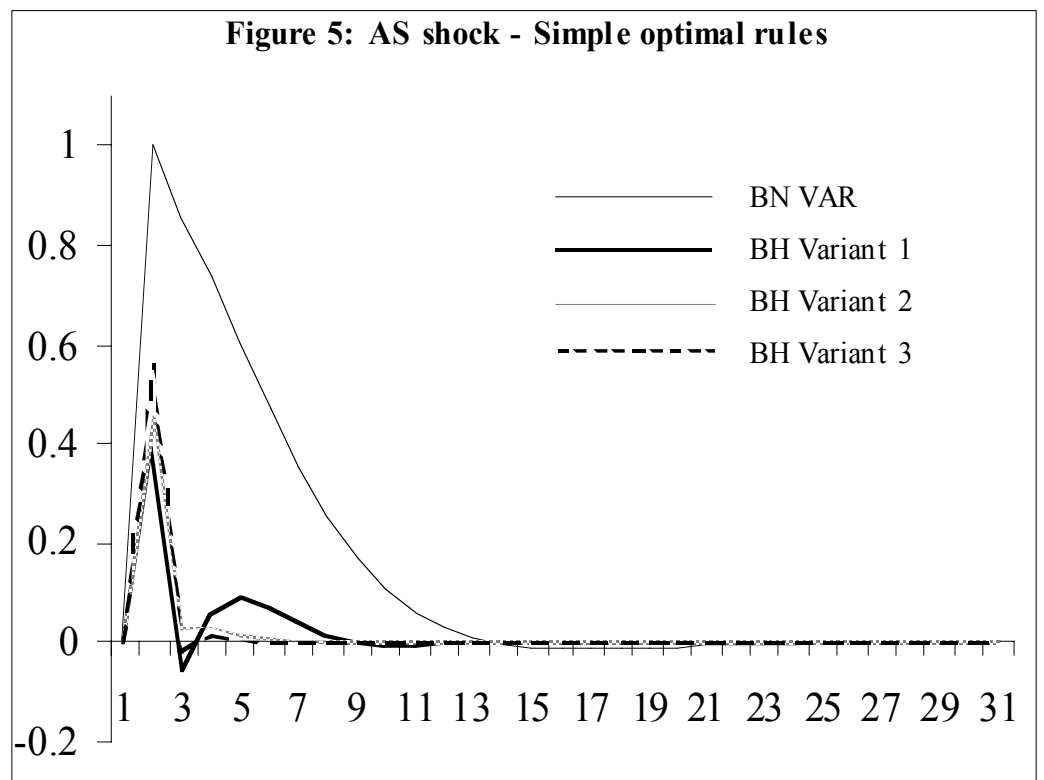

Figure 5 depicts the impulse responses of inflation for the four models in reaction to a temporary 1\% shock to aggregate supply. Qualitatively, Figure 5's plots for the simple optimal rule are similar to those for the fully optimal rule in Figure 2: the three variants of the $\mathrm{BH}$ model feature a policy rule which prevents the aggregate supply shock from appearing one-for-one in the inflation rate, while in the VAR the $1 \%$ shock does produce an initial commensurate increase in inflation because policy can affect inflation only with a lag. Even in the $\mathrm{BH}$ variants, however, the peak of inflation above target exceeds 0.3 percent, whereas it was below 0.3 for all variants in Figure 2 . This poorer performance reflects the movement from a fully optimal policy to a restrained-optimal policy. As far as the time it takes for inflation to get back on target is concerned, the results for all models in Figure 5 are similar to those under the fully optimal policy, so we refer the reader to the earlier discussion. 


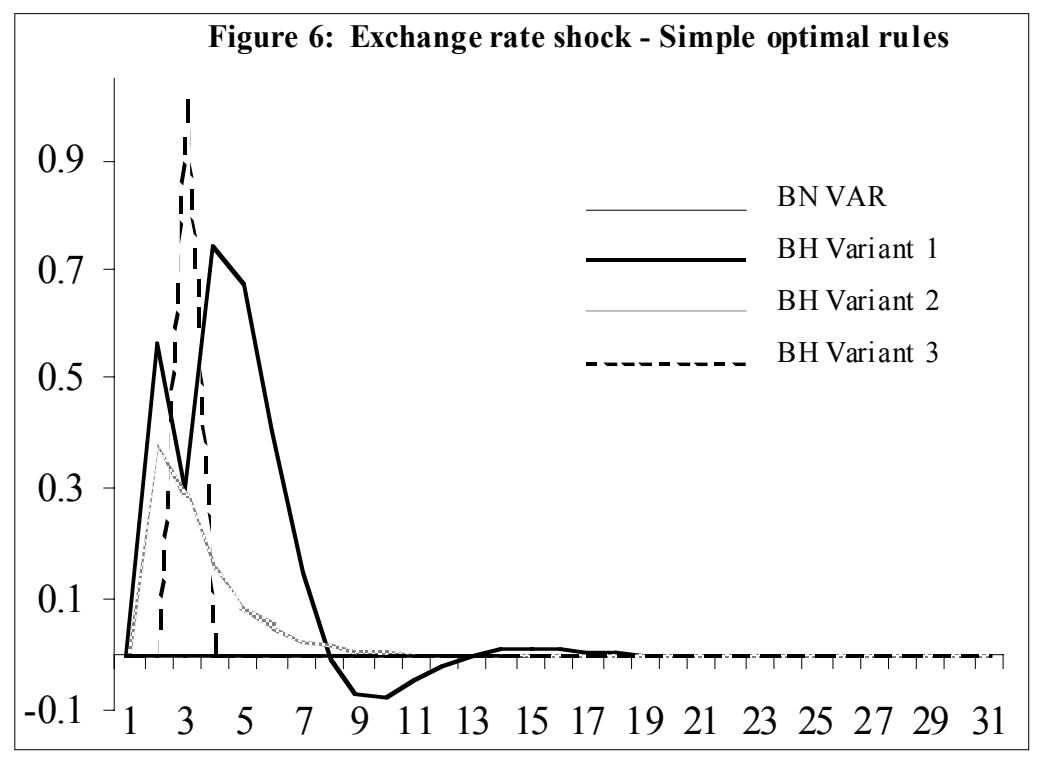

Figure 6 displays the impulse responses of inflation for the four models, following a temporary $1 \%$ real exchange rate shock. In line with our earlier results, the insignificance of the exchange rate in the VAR means that the impulse response is flat for this model. As for the $\mathrm{BH}$ variants, the difference between a fully optimal and a 'simple optimal' rule seems to be quite strong in the case of an exchange rate shock. The peak effect of the exchange rate shock on inflation is $0.73 \%$ (Variant 1 ), $0.28 \%$ (Variant 2), and $1 \%$ (Variant 3$)$, compared to $(0.55 \%, 0.38 \%,-0.39 \%)$ respectively in Figure 3 under the fully optimal rule. The reason why the result is so different for $\mathrm{BH}$ Variant 3 is as follows: under simple rule (3.1), $R_{t}$ is a predetermined variable so it is powerless to offset contemporaneous exchange rate innovations. Therefore, a $1 \%$ shock to $\varepsilon_{U I P t}$ in equation (1.5) shocks the right hand side of (1.4), i.e. $\mathrm{E}_{t} \Delta q_{t+1}+\mathrm{E}_{t} \pi_{t+1}$, by the same amount — and both these variables strongly affect inflation in $\mathrm{BH}$ Variant 3.

However, while Figure 6 indicates that the deviations of inflation from target are greater, the period of time before inflation settles back on target is somewhat shorter under the simple rules than it was under the fully optimal rules of Figure 3. 
The figures above provide prima facie evidence about the nature — and length — of (non-optimal) policy horizons as implied by the optimised simple feedback rules. However, to answer more fully the initial question of what policy horizons are implied by simple feedback optimal rules, we need to present policy horizons computed for each model under the various shocks for all criteria; we do this next. For simplicity, we divide the analysis into two parts. First, we study whether feeding back on inflation at some horizon $k$ periods ahead implies also taking inflation back to target at that horizon (in other words, is the $\mathrm{OFH}$ equal to the $\mathrm{PH}$ implied by the simple optimal rule that delivers that OFH?); secondly, we investigate whether OPHs and PHs associated with OFHs differ. That is, we ask: does the speed of disinflation implied by simple optimal and fully optimal rules differ, and if so, how?

We address the first part of the analysis by looking at Tables 15-17. These compare optimal $k \mathrm{~s}$ under the 'feedback horizon' interpretation of horizon (OFHs) with the non-optimal $k$ s (in their various definitions) under the 'policy horizon' (speed of disinflation) interpretation of horizon. Recall that the OFH is, by definition, the value of $k$ optimally chosen when policy is constrained to feed back merely on the deviation of inflation expected $k$ periods hence from the target; and that the optimality criterion according to which an OFH is chosen consists of minimisation of the loss function (1.1) in stochastic simulations. Consequently, the $\mathrm{OFH}$ is not a $k$ that varies depending on the shock type, and therefore, for each model, $k^{*}(\mathrm{OFH})$ is the same across Tables 15 to $17 .^{9}$

\footnotetext{
${ }^{9}$ Essentially, the criterion used for deriving the OFH makes us select the optimal $k$ in light of the presence of all three shocks simultaneously in stochastic simulations, with the importance of each shock depending both on how strongly it enters the model's equations and on the shock variance-
} 


\begin{tabular}{|l|c|c|c|c|}
\hline \multicolumn{5}{|c|}{ Table 15: OFH and PH comparison - AD shock } \\
\hline & $k^{*}(\mathrm{OFH})$ & $k_{A 1}(\mathrm{PH})$ & $k_{A 2}(\mathrm{PH})$ & $k_{R}(\mathrm{PH})$ \\
\hline BN VAR & 9 & 0 & 14 & 16 \\
\hline BH Variant 1 & 2 & 1 & 8 & 8 \\
\hline BH Variant 2 & 4 & 0 & 4 & 7 \\
\hline BH Variant 3 & 1 & 0 & 4 & 5 \\
\hline
\end{tabular}

\begin{tabular}{|l|c|c|c|c|}
\hline \multicolumn{5}{|c|}{ Table 16: OFH and PH comparison — AS shock } \\
\hline & $k^{*}(\mathrm{OFH})$ & $k_{A 1}(\mathrm{PH})$ & $k_{A 2}(\mathrm{PH})$ & $k_{R}(\mathrm{PH})$ \\
\hline BN VAR & 9 & 1 & 11 & 11 \\
\hline BH Variant 1 & 2 & 0 & 6 & 7 \\
\hline BH Variant 2 & 4 & 0 & 2 & 4 \\
\hline BH Variant 3 & 1 & 0 & 2 & 2 \\
\hline
\end{tabular}




\begin{tabular}{|l|c|c|c|c|}
\hline \multicolumn{5}{|c|}{ Table 17: OFH and PH comparison, exchange rate shock } \\
\hline & $k^{*}(\mathrm{OFH})$ & $k_{A 1}(\mathrm{PH})$ & $k_{A 2}(\mathrm{PH})$ & $k_{R}(\mathrm{PH})$ \\
\hline BN VAR & 9 & 0 & 0 & 13 \\
\hline BH Variant 1 & 2 & 0 & 11 & 11 \\
\hline BH Variant 2 & 4 & 0 & 5 & 7 \\
\hline BH Variant 3 & 1 & 0 & 3 & 3 \\
\hline
\end{tabular}

Two main points emerge from the tables above. First, the number of periods ahead associated with the value of expected inflation that it is optimal to feed back upon $\left(k^{*}\right.$ $(\mathrm{OFH})$ ) does not coincide with the time at which inflation settles on target when policy is actually set in response to expected inflation deviations $k$ periods ahead (i.e., for each Table, horizons in the first column do not coincide with horizons in the following columns). Thus, if policymakers employ a simple rule, it may be optimal to focus on forecasts of inflation for a certain time in the future; but this does not imply stabilising inflation in that future period. Second, and on a closely related point, the time in the future whose expected inflation rate is best to use in the feedback rule is generally shorter than the horizon at which inflation is back on target under that same simple rule. ${ }^{10}$ For example, for BH Variant 1 , feeding back on the deviation of inflation expected at $k=2$ periods ahead as implied by the optimisation of parameters in simple rule (3.2), implies that inflation reaches the target only at $k=8$ (18 months later than the time in the future associated with the inflation forecast in the feedback rule). Put differently, if policymakers wish to take inflation back to target two years 
after a shock using a simple rule, they apparently should feed back on two-quarterahead expected inflation (relative to target). ${ }^{11}$

We now address the second part of the analysis and investigate how the above (nonoptimal) policy horizons relate to their corresponding (same model, loss function, and shock) optimal policy horizons of Section 2. Tables 18-21 list the (non-optimal) policy horizons associated with their respective OFHs under the three criteria, alongside the optimal policy horizons of Section 2.

For the BN VAR (Table 18), the non-optimal (non-starred) policy horizon $k_{A 2}$ differs by four quarters from its optimal counterpart $k_{A 2}{ }^{*}$, but it is still in the $2_{-}$years range. So, even if $k_{A 2}$ and $k_{A 2} *$ differ by a full year, they are qualitatively in agreement in suggesting returning inflation back to target over the medium term ( 2 to 4 years) rather than over the short term (1 quarter to 1 year). This is true both of the aggregate demand and the aggregate supply shocks (the absolute criterion $k_{A 2}$ suggests 11 quarters for simple optimal rules $\left(k_{A 2}\right)$ and 16 for the optimal rule $\left.\left(k_{A 2}{ }^{*}\right)\right)$. Similarly, the essential irrelevance of the exchange rate in the estimated VAR again means that $k$ is trivially zero under both simple and fully optimal rules. As for the relative criterion, the non-optimal policy horizon $\left(k_{R}\right)$ for the $\mathrm{AD}$ shock is the same (16 quarters) as the optimal policy horizon $\left(k_{R}^{*}\right)$; and non-optimal and optimal policy horizons are qualitatively similar (both ranging between 3 and 4 years) for aggregate supply and exchange rate shocks.

In summary, regardless of whether one considers non-optimal horizons (PHs) or optimal policy horizons (OPHs), and regardless of the criterion used, the VAR supports a speed of disinflation of 3 to 4 years in response to demand and supply

\footnotetext{
${ }^{11}$ And, correspondingly, the table suggests feeding back on deviations of inflation expected nine quarters ahead to take inflation back to target in fourteen quarters for the BN VAR after an AD shock; and feeding back on one-period-ahead expected inflation to bring inflation back on target a year after a shock in BH Variant 3. Interestingly, BH Variant 2 is the only model for which the exercise suggests that inflation would be on target at the same time at which policymakers form a forecast of inflation on whose deviations from target they actually feed back from $(k=4$ is both the optimal feedback horizon
} 
shocks. ${ }^{12}$ This reflects the protracted estimated dynamics of the BN VAR model. All this has to be subject to the caveat that the optimised feedback parameter that governs the inflation path under the simple optimal rules ( $\mathrm{PH}$ case) is very large, reducing the realism of the policy rule contemplated.

In the case of BH Variant 1 (Table 19) - i.e. the BH model with a backward-looking IS function and Fuhrer- Moore contracting, with a weight of 0.8 on the backwardlooking component of inflation expectations in the augmented Phillips curve - the policy horizon $k_{A 2}$ under the aggregate demand shock suggests that 8 quarters ahead is the best time at which to stabilise inflation back on target; and similarly, eight quarters for the policy horizon under the relative criterion. For the exchange rate shock, regardless of the criterion adopted, the $\mathrm{PH}$ and $\mathrm{OPH}$ are broadly similar, proposing to bring inflation on target in about 3 to 5 years.

Results for BH Variants 2 and 3 are given in Tables 20 and 21 respectively. Reflecting their more forward-looking nature, Variant 2 (forward-looking IS curve, Fuhrer-Moore price contracting) and Variant 3 (backward-looking IS curve but fully forward-looking, Taylor-style, price contracting) tend to deliver, on average, lower values of $k$. For example, in Table 17 (BH Variant 2), after a demand shock, inflation is on target according to the various criteria within 2 years (more precisely within 4 to 7 quarters in the $\mathrm{PH}$ case and within 2 to 7 quarters in the $\mathrm{OPH}$ case) compared with $(8,8)$ and $(10,10)$ quarters of the BH Variant 1.

Likewise, the impact on inflation of the supply shock is reined in much faster in $\mathrm{BH}$ Variant 2 than in BH Variant 1, regardless of whether we use an optimised simple or a fully optimal rule. Almost the same conclusions apply if $\mathrm{BH}$ Variant 3 is considered.

${ }^{12}$ This is, of course, ignoring results from the first absolute criterion $k_{A 1}$ and/or $k_{A 1}{ }^{*}$, for the reasons 
Table 18: Policy Horizons - $\mathrm{BN} \mathrm{VAR}^{a}$

Weight combination in the loss function $\lambda \pi=\lambda_{y}=0.5$

\begin{tabular}{|c|c|c|c|c|c|c|}
\hline & \multicolumn{4}{|c|}{ Policy horizons } & \multicolumn{2}{c|}{ Optimal policy horizons } \\
(ex Section 2)
\end{tabular}

$(a): k_{A 1} *$ refers to the number of periods, under the optimal rule, that it takes inflation to return permanently to within $+/-1$ percent of its initial value, following an initial 1 percent shock of the indicated type; $k_{A 2} *$ refers to the number of periods, under the optimal rule, that it takes inflation to return permanently to within 0.05 percent of its initial value, following an initial 1 percent shock of the indicated type; $k_{R}{ }^{*}$ refers to the number of periods it takes for 95 percent of the initial effect of the indicated shock to inflation to have been permanently eroded.

\begin{tabular}{|c|c|c|c|c|c|c|}
\hline \multicolumn{7}{|c|}{$\begin{array}{l}\text { Table 19: Policy Horizons }- \text { BH Variant } 1^{a} \\
\text { Weight combination in the loss function } \lambda_{\pi}=\lambda_{y}=0.5\end{array}$} \\
\hline & \multicolumn{3}{|c|}{ Policy horizons } & \multicolumn{3}{|c|}{$\begin{array}{l}\text { Optimal policy horizons } \\
\text { (ex Section } 2)\end{array}$} \\
\hline & $k_{A 1}$ & $k_{A 2}$ & $k_{R}$ & $k_{A 1} *$ & $k_{A 2}{ }^{*}$ & $k_{R}^{*}$ \\
\hline AD shock & 1 & 8 & 8 & 1 & 10 & 10 \\
\hline AS shock & 0 & 6 & 7 & 0 & 3 & 11 \\
\hline Exch. rate shock & 0 & 11 & 11 & 0 & 13 & 20 \\
\hline \multicolumn{7}{|c|}{$\begin{array}{l}\text { (a): BH Variant } 1 \text { has a backward-looking IS function and a mixed backward / forward-looking AS } \\
\text { equation. } k_{A 1} * \text { refers to the number of periods, under the optimal rule, that it takes inflation to return } \\
\text { permanently to within }+/-1 \text { percent of its initial value, following an initial } 1 \text { percent shock of the } \\
\text { indicated type; } k_{A 2} * \text { refers to the number of periods, under the optimal rule, that it takes inflation to } \\
\text { return permanently to within } 0.05 \text { percent of its initial value, following an initial } 1 \text { percent shock of the } \\
\text { indicated type; } k_{R}^{*} \text { refers to the number of periods it takes for } 95 \text { percent of the initial effect of the } \\
\text { indicated shock to inflation to have been permanently eroded. }\end{array}$} \\
\hline
\end{tabular}


Table 20: Policy Horizons - BH Variant $2^{a}$

Weight combination in the loss function $\lambda \pi=\lambda_{y}=0.5$

\begin{tabular}{|c|c|c|c|c|c|c|}
\hline & \multicolumn{3}{|c|}{ Policy horizons } & \multicolumn{3}{c|}{ Optimal policy horizons } \\
(ex Section 2)
\end{tabular}

(a): BH Variant 2 has a forward-looking IS function and a mixed backward / forward-looking AS equation. $k_{A 1} *$ refers to the number of periods, under the optimal rule, that it takes inflation to return permanently to within $+/-1$ percent of its initial value, following an initial 1 percent shock of the indicated type; $k_{A 2} *$ refers to the number of periods, under the optimal rule, that it takes inflation to return permanently to within 0.05 percent of its initial value, following an initial 1 percent shock of the indicated type; $k_{R}{ }^{*}$ refers to the number of periods it takes for 95 percent of the initial effect of the indicated shock to inflation to have been permanently eroded.

\begin{tabular}{|c|c|c|c|c|c|c|}
\hline \multicolumn{7}{|c|}{$\begin{array}{l}\text { Table 21: Policy Horizons }- \text { BH Variant } 3^{a} \\
\text { Weight combination in the loss function } \lambda_{\pi}=\lambda_{y}=0.5\end{array}$} \\
\hline & \multicolumn{3}{|c|}{ Policy horizons } & \multicolumn{3}{|c|}{$\begin{array}{l}\text { Optimal policy horizons } \\
\text { (ex Section } 2)\end{array}$} \\
\hline & $k_{A 1}$ & $k_{A 2}$ & $k_{R}$ & $k_{A 1} *$ & $k_{A 2}{ }^{*}$ & $k_{R}^{*}$ \\
\hline AD shock & 0 & 4 & 5 & 0 & 10 & 12 \\
\hline AS shock & 0 & 2 & 2 & 0 & 3 & 18 \\
\hline Exch. rate shock & 0 & 3 & 3 & 0 & 10 & 18 \\
\hline \multicolumn{7}{|c|}{$\begin{array}{l}\text { (a): BH Variant } 3 \text { has a backward-looking IS function and a forward-looking AS equation. } k_{A 1} * \text { refers } \\
\text { to the number of periods, under the optimal rule, that it takes inflation to return permanently to within } \\
+/-1 \text { percent of its initial value, following an initial } 1 \text { percent shock of the indicated type; } k_{A 2}{ }^{*} \text { refers to } \\
\text { the number of periods, under the optimal rule, that it takes inflation to return permanently to within } \\
0.05 \text { percent of its initial value, following an initial } 1 \text { percent shock of the indicated type; } k_{R}{ }^{*} \text { refers to } \\
\text { the number of periods it takes for } 95 \text { percent of the initial effect of the indicated shock to inflation to } \\
\text { have been permanently eroded. }\end{array}$} \\
\hline
\end{tabular}




\section{Conclusions}

In this paper we investigated the problem of selecting an optimal horizon for inflation targeting in the United Kingdom.

There are various ways to think of an 'optimal horizon' for inflation targeting. One way is to think of it as the best $k$ when the authorities should feed back on expected inflation $k$ periods ahead (using a simple rule). A second way is to think of it as the time at which inflation is restored to target when the authorities aim at minimising the costs of disinflation (i.e., use an optimal control rule for the instrument). We referred to the first kind of horizon as the optimal feedback horizon (OFH) and to the second kind as the optimal policy horizon $(\mathrm{OPH})$.

In our analysis above, we computed optimal policy and feedback horizons numerically. To see whether our results are robust in the face of model uncertainty, we derived results for a set of models with varying structural and dynamic characteristics. In particular, we derived results for two different models of the UK economy: a vector autoregression (VAR) estimated on quarterly data; and several variants of a small calibrated forward-looking model (Batini and Haldane (1999)).

Our findings can be summarised as follows.

Results on optimal policy horizons (OPHs)

While our results on optimal policy horizons are model-dependent and vary with the shock under consideration, there are several common elements to them when grouped by type of disturbance.

\section{This partof the paper is being revised (sensitive)}


Results on optimal feedback horizons (OFHs)

This part of the project investigated the inflation forecast horizon $k$ on which it is optimal for the authorities to feed back when employing a simple explicit policy rule of the form:

$R_{t}=\rho_{R} R_{t-1}+\psi_{p}\left(\mathrm{E}_{t-1} \pi_{t+k}-\pi^{T A R}\right)$

where the notations is as described at the beginning of Section 3 .

We found that:

This part of the paper is being revised (ibidem)

What do our results imply for policymakers?

This part of the paper is being revised (ibidem) 


\section{Data Appendix}

Definition and sources of the variables used in the BN VAR

The variables we use in the VAR are:

$y_{t}: \log$ of real GDP (quarterly) new ONS national accounts at constant factor costs, with the estimated quadratic trend for 1971:3-1998:2 removed.

$\pi_{t}^{D \mathrm{E} V}: \pi_{t}-\pi_{t}^{T A R}$, where:

$\pi_{t}: \log \left(R P I X_{t} / R P I X_{t-4}\right)$, where $R P I X_{t}$ is the RPIX deflator in quarter $t$. Our use of the four-quarter inflation rate rather than the quarterly change is motivated by the fact that, historically, targets for UK inflation or other nominal aggregates have been expressed in terms of annual changes rather than quarter-to-quarter movements. It is also conceivable that a four-quarter inflation rate may be a better empirical measure of underlying quarterly inflation than actual quarterly inflation.

$\pi_{t}^{\text {TAR }}$ : Target (annualised) inflation rate, calculated as follows:

-1976:3-1985:1: The implicit inflation target was the one implied by the £M3 targets. Using the assumption about trend velocity growth of $+1.25 \%$ per annum stated by the Treasury in $1980^{13}$, we backed out the nominal income growth targets implicit in the 1976-1980 £M3 targets and in the targets for 1980-1984 announced in the Medium Term Financial Strategy (MTFS) in 1980. To obtain the implied inflation target, we subtracted $21 / 2$ percent (to allow for output growth) from each nominal income growth target. 
A new set of $£$ M3 targets for 1982-1985 were announced in March 1982; for the years also covered by the 1980 MTFS, the $£$ M3 growth targets now had a 3 percentage point higher midpoint, which we take as a change in the annual trend velocity growth assumption from $+1.25 \%$ to $-1.75 \%$ rather than changed targets for nominal income growth. Subsequent revisions to the 1982 MTFS are again assumed to represent changed assumptions about velocity rather than changed targets for nominal income growth.

From 1985 to 1990, we use the announced nominal income growth target, then subtract 2.5 percent for real growth.

-1990:4-1992:3: given the ERM agreement, the implicit inflation target coincided with the German 2\% inflation target;

- 1992:4 onwards: 2.5\% (explicit inflation target).

$\Delta e_{t}$ : Nominal exchange rate change, $\log \left(E R I_{t} / E R I_{t-1}\right)$, where $E R I$ is the Exchange Rate Index. This variable is measured such that an observation of -0.10 indicates a depreciation of sterling of $10 \%$.

$R_{t}$ : Quarterly average of the annualised nominal interbank lending rate, measured as a fraction.

\section{Time Series Properties of the Data}

We now show that all of the variables in our VAR are adequately described as stationary $(\mathrm{I}(0))$ or trend-stationary processes, and hence, a Johansen-style cointegration approach to our VAR is not appropriate.

\footnotetext{
${ }^{13}$ The Treasury, in its submission to the Treasury and Civil Service Committee (1980), estimated
} 
During our sample period, output $\left(y_{t}\right)$ can be modelled as trend-stationary, with $\pi_{t}^{D \mathrm{E} V}$ and $\Delta e_{t}$ treated as $\mathrm{I}(0)$ series, and $R_{t}$ as an $\mathrm{I}(0)$ series after controlling for key shifts in monetary policy regime. As evidence, in Table 1A we present Augmented Dickey Fuller (ADF) statistics that test the null of a unit root for the variables in our model. Since our contention is that output is trend-stationary, Table 1A actually gives a test for a unit root in detrended output. ${ }^{14}$ Two ADF test statistics are calculated for the nominal interest rate, $R_{t}$ : the first includes only a constant in the ADF regression, whereas the second includes a constant, $D E R M_{t}$, and $D 924_{t}$. Excluding these dummy variables may bias the test toward suggesting a unit root in $R_{t}{ }^{15}$

Table 1A: $\quad$ ADF Tests for Batini-Nelson VAR

\begin{tabular}{|c|c|}
\hline Variable & ADF statistic \\
\hline Detrended $y_{t}$ & $-2.427^{*}$ \\
\hline$\pi_{t}^{D \mathrm{E} V}$ & $-3.297^{*}$ \\
\hline$\Delta e_{t}$ & $-3.817^{* *}$ \\
\hline$R_{t}$ (no shifts) & -1.984 \\
\hline$R_{t}$ (shifts included) & $-3.675^{*}$ \\
\hline
\end{tabular}

Note: A lag length of four is used in the ADF regressions for each variable except $R_{t}$ (one lag). A * denotes significance at 0.05 level according to the Dickey-Fuller distribution's critical values; a ** significance at the 0.01 level according to these values.

The tests reject the null of a unit root in favour of the alternative of stationarity (or, in the case of $R_{t}$, an I(0) series with structural breaks). Thus, the elements of our VAR are all $\mathrm{I}(0)$, and no testing for or estimation of cointegrating vectors is required. ${ }^{16}$

trend £M3 velocity growth at 1.0 to $1.5 \%$ per annum.

${ }^{14}$ Detrended output is the residual from a prior regression of $y_{t}$ on a constant and linear trend over 1980Q3-1998Q1. No constant is included in the regression used in calculating the ADF test for the stationarity of detrended output because the dependent variable is mean zero by construction.

${ }^{15}$ A one-time step-change in the intercept of an $\mathrm{I}(0)$ series, even if small, can lead to that series appearing to be I(1) [Hendry and Neale (1991, p. 96)].

${ }^{16}$ There is also an economic reason for not favouring a cointegration-based approach to this VAR. The only real variable in the VAR is $y_{t}$ and we do not want to explain the trend in $y_{t}$ with nominal 


\section{Technical Appendix - Solution Methods}

Consider first the policymaker's problem for determining optimal policy when the economy's structure is described by the VAR equations (V1)-(V3). If we let $\Lambda_{t}, \phi_{t}$, and $\Omega_{t}$ denote the Lagrange multipliers on (V1)-(V3) respectively, the policymaker's first order conditions (with respect to $y_{t}, \pi_{t}, \Delta e_{t}$, and $R_{t}$ ) for minimising the objective function (1) are:

$\beta a_{11} \mathrm{E}_{t} \Lambda_{t+1}+\beta b_{11} \mathrm{E}_{t} \phi_{t+1}+\beta c_{11} \mathrm{E}_{t} \Omega_{t+1}=2 w_{2} y_{t}+\Lambda_{t}$

$\beta a_{12} \mathrm{E}_{t} \Lambda_{t+1}+\beta b_{12} \mathrm{E}_{t} \phi_{t+1}+\beta c_{12} \mathrm{E}_{t} \Omega_{t+1}=2 w_{1} \pi_{t}+\phi_{t}$

$\beta a_{13} \mathrm{E}_{t} \Lambda_{t+1}+\beta b_{13} \mathrm{E}_{t} \phi_{t+1}+\beta c_{13} \mathrm{E}_{t} \Omega_{t+1}=\Omega_{t}$

$\beta a_{14} \mathrm{E}_{t} \Lambda_{t+1}+\beta b_{14} \mathrm{E}_{t} \phi_{t+1}+\beta c_{14} \mathrm{E}_{t} \Omega_{t+1}=0$

Following King and Wolman (1999), we solve for the optimal policy rule by writing the problem as a system of linear expectational difference equations. The VAR equations (V1)-(V3) for $y_{t}, \pi_{t}$, and $\Delta e_{t}$, combined with the policymaker's first order conditions (A1)-(A4) for optimal policy, can be cast in the first-order form [as in King and Watson (1995)]:

$\boldsymbol{A} \mathrm{E}_{t} \mathbf{y}_{t+1}=\boldsymbol{B} \mathbf{y}_{t}+\boldsymbol{C} \mathbf{z}_{t}$

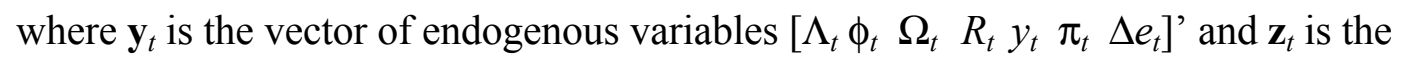
vector of exogenous shocks.

In solving for optimal policy, we suppress the shocks to equations (V1)-(V3), in effect exploiting certainty equivalence. We end up with a rule of the form 
$R_{t}=\phi_{Y} y_{t}+\phi \pi \pi_{t}+\phi_{e} \Delta e_{t}$. Then for stochastic simulation and the calculation of impulse responses under this rule, we restore the shocks to (V1)-(V3).

The corresponding optimisation problem for the Batini-Haldane (1999) model is not a standard optimal control problem because the model's equations, and therefore the policymaker's constraints, contain forward-looking elements. A similar problem is faced by King and Wolman (1999), who assume certainty equivalence and then follow the standard Lagrangean optimisation approach. We follow King and Wolman's procedure here. As in their paper, the presence of forward-looking constraints has the effect of putting lagged Lagrange multipliers in the state vector. Consequently, the policymaker's optimal rule for $R_{t}$ is a linear function of the vector $\left[y_{t} \phi_{t-1} \Lambda_{t-1} \Omega_{t-1} \pi_{t-1} q_{t-1} y_{t-1}\right]$ ', where $q_{t-1}, \Lambda_{t-1}$, and $\Omega_{t-1}$ are, respectively, the Lagrange multipliers on (1.3) and the one-period-lagged versions of (1.4) and (1.5). 


\section{References}

Coletti et al (1996)

Batini, N and Haldane, A G (1999). 'Forward-Looking Rules for Monetary Policy'. In

John B. Taylor (ed.), Monetary Policy Rules. University of Chicago Press for NBER.

Black, R, Macklem, T and Rose, D (1997), “On Policy Rules for Price Stability”, in Price Stability, Inflation Targets and Monetary Policy, Bank of Canada.

Bowen, A (1995) 'Inflation targetry in the United Kingdom'. In A.G. Haldane (1995)

Targeting Inflation. Bank of England. 59-74..

Brainard, W (1967), 'Uncertainty and the effectiveness of policy', American Economic Review, 52,

de Brouwer, J and O’Regan, J (1997), “Evaluating Simple Monetary-Policy Rules for Australia", in P Lowe (ed.), Monetary Policy and Inflation Targeting, Reserve Bank of Australia.

Fuhrer, J C (1997), 'The (Un)importance of Forward-Looking Behaviour in Price Specifications', Journal of Money, Credit, and Banking, 29, 338-350.

Fuhrer, J C and Moore G (1995). 'Inflation Persistence', Quarterly Journal of Economics, 110, 127-159.

Haldane, A G (ed.) (1995). Targeting Inflation. Bank of England.

Hendry, D F., and Neale, A J (1991). "A Monte Carlo Study of the Effects of Structural Breaks on Tests for Unit Roots". In Hackl, P and Westlund A H (eds.), Economic Structural Change, Analysis and Forecasting. Springer Verlag. 95-119. 
HM Treasury (1980). 'The Stability of the Income Velocity of Circulation of Money Supply'. In Third Report from the Treasury and Civil Service Committee, Session 1980-81: Monetary Policy, Volume II: Minutes of Evidence. HM Stationery Office. 126-129.

King, M A (1996). 'Direct Inflation Targets'. Paper presented at Geldpolitische in Europe symposium, Frankfurt.

King, R G and Watson, M W (1995), 'The Solution of Singular Linear Difference Systems Under Rational Expectations', manuscript, University of Virginia. Forthcoming in International Economic Review.

King, R G, and Wolman A L (1999), 'What Should the Monetary Authority Do When Prices are Sticky?'. In John B. Taylor (ed.), Monetary Policy Rules. University of Chicago Press for NBER.

McCallum, B T and Nelson E (1999), ‘An Optimising IS-LM Specification for Monetary Policy and Business Cycle Analysis', Journal of Money, Credit, and Banking, forthcoming.

Nixon, J and Hall, S (1996) 'Controlling Inflation: Modelling Monetary Policy in the 1990s', CEF, London Business School, Discussion Paper No. DP 4-96.

Rotemberg, J J, and Woodford, M (1999). 'Interest Rate Rules in an Estimated Sticky-Price Model'. In J B Taylor (ed.), Monetary Policy Rules. University of Chicago Press for NBER.

Svensson, L E O (1996), "Price Level Targeting Versus Inflation Targeting: A Free Lunch?”, NBER Working Paper No.5719. 
Svensson, L E O (1997a), "Inflation-Forecast-Targeting: Implementing and Monitoring Inflation Targets”, European Economic Review, Vol.41, 1111-46.

Taylor, J B (1980). ‘Aggregate Dynamics and Staggered Contracts', Journal of Political Economy, 88, 1-23.

Taylor, J B (ed.) (1999). Monetary Policy Rules. University of Chicago Press for NBER. 